\title{
Cellular localization of NRF2 determines the self-renewal and osteogenic differentiation potential of human MSCs via the P53-SIRT1 axis
}

\author{
DS Yoon ${ }^{1}$, Y Choi ${ }^{1,2}$ and JW Lee ${ }^{*, 1,2}$
}

NRF2 (nuclear factor erythroid-derived 2-like 2) plays an important role in defense against oxidative stress at the cellular level. Recently, the roles of NRF2 in embryonic and adult stem cells have been reported, but its role in maintaining self-renewal and differentiation potential remains unknown. We studied the mechanisms of NRF2 action in mesenchymal stem cells (MSCs) derived from human bone marrow. We found that the cellular localization of NRF2 changed during prolonged cell passage and osteogenic differentiation. Blocking the nuclear import of NRF2 using ochratoxin A (OTA) induced the loss of the self-renewal and osteogenic potential of early-passage (EP) MSCs. Conversely, reinforcing the nuclear import of NRF2 using tert-butylhydroquinone (t-BHQ) improved the self-renewal capacity and maintained the differentiation potential in the osteogenic lineage of EP MSCs. Real-time quantitative PCR and western blot analysis showed that NRF2 positively regulates sirtuin 1 (SIRT1) at the mRNA and protein levels via the negative regulation of p53. The self-renewal and osteogenic potential suppressed in OTA-treated or NRF2-targeting small hairpin RNA (shRNA)-infected EP MSCs were rescued by introducing small interfering RNA (siRNA) targeting p53. t-BHQ treatment in late-passage (LP) MSCs, which lost their self-renewal and osteogenic potential, reversed these effects. In LP MSCs treated with t-BHQ for $\sim 7$ days, the phosphorylation and nuclear localization of NRF2 improved and SIRT1 protein level increased, whereas p53 protein levels decreased. Therefore, our results suggest that NRF2 plays an important role in regulating p53 and SIRT1 to maintain MSC stemness. This study is the first to establish a functional link between NRF2 and SIRT1 expression in the maintenance of MSC self-renewal and differentiation potential.

Cell Death and Disease (2016) 7, e2093; doi:10.1038/cddis.2016.3; published online 11 February 2016

Mesenchymal stem cells (MSCs) grown in ex vivo conditions become senescent during prolonged cell passage. Consequently, long-term cultured stem cells are not used for tissue regeneration because they do not function as stem cells after reaching therapeutically effective amounts during in vitro cultivation. Appropriate culture methods are very important to maintain MSC stem cell potential because their self-renewal and multipotency decrease during ex vivo cultivation. ${ }^{1-5}$ The decrease in the self-renewal and differentiation potentials during ex vivo conditions may be related to exposure to exogenous oxidative stress or to reactive oxygen species (ROS) that are endogenously generated in in vitro cultured cells. ${ }^{6,7}$ Hypoxic conditions have been extensively examined with respect to the maintenance of the self-renewal and multipotency of human MSCs during long-term cultivation. ${ }^{8-11}$ Indeed, in vivo MSC niches where the cells reside exhibit low-oxygen tension (2-8\%). ${ }^{12}$ A recent study has also reinforced the importance of hypoxic conditions for the maintenance of MSC stemness. ${ }^{13}$ Therefore, hypoxic conditions may be essential for the maintenance of the early characteristics of ex vivo cultured MSCs. In hypoxic environments, the osteogenic and adipogenic potentials of human bone marrow-derived MSCs (BM-MSCs) decrease. ${ }^{13}$ However, their self-renewal capacity is maintained. ${ }^{14,15}$ These results indicate that hypoxic environments in MSC cultivation maintain the undifferentiated state of MSCs without affecting their differentiation potential, promoting stemness over differentiation. However, no studies have examined the master regulators related to the maintenance of stemness in MSC cultivation in hypoxic environments in vitro.

Nuclear factor erythroid-derived 2-like 2 (NRF2) plays an important role in defense against oxidative stresses. ${ }^{16,17}$ It induces the expression of antioxidant genes that protect many cell types from oxidative stress caused by tissue damage, such as inflammation and injury. ${ }^{18}$ Under normal condition, NRF2 remains in the cytoplasm, and is degraded via ubiquitination triggered by binding with kelch like-ECHassociated protein 1 (KEAP1). ${ }^{19}$ Under oxidative stress, NRF2 is phosphorylated and moves from the cytoplasm to the nucleus by disrupting the complex with KEAP1. ${ }^{20,21}$ In this regard, the regulation of NRF2 cellular localization may be important to overcome MSC aging during prolonged cell

\footnotetext{
${ }^{1}$ Department of Orthopaedic Surgery, Yonsei University College of Medicine, Seoul, South Korea and ${ }^{2}$ Brain Korea 21 PLUS Project for Medical Science, Yonsei University, Seoul, South Korea

*Corresponding author: JW Lee, Department of Orthopedic Surgery-29, Yonsei University College of Medicine, 250, Seongsanno, Seodaemun-gu, Seoul 120-752, South Korea. Tel: +82 22228 2190; Fax: +82 2363 1139; E-mail: ljwos@yuhs.ac

Abbreviations: BM-MSC, bone marrow-mesenchymal stem cell; ROS, reactive oxygen species; NRF2, nuclear factor erythroid-derived 2-like 2; CFU-F, colony-forming unit fibroblast; DAPI, 4,6-diamidino-2-phenyindole; FITC, fluorescein isothiocyanate; t-BHQ, tert-butylhydroquinone; HO-1, heme oxygenase-1; NQO-1, NAD(P)H dehydrogenase (quinone 1); OTA, ochratoxin A; RUNX2, Runt-related transcription factor 2; SIRT1, sirtuin 1; siRNA, small interfering RNA; HIC1, hypermethylated in cancer 1

Received 23.9.15; revised 18.11.15; accepted 23.12.15; Edited by Y Shi
} 
passages in vitro because oxidative stress induces premature senescence of MSCs. ${ }^{22}$ NRF2 overexpression protects MSCs against cell death and apoptosis caused by oxidative stress and retains multi-differentiation potential. ${ }^{23} \mathrm{~A}$ recent study has shown that NRF2 controls the self-renewal and pluripotency of human embryonic stem cells. ${ }^{24}$ These results imply that NRF2 may regulate MSC stemness. However, no studies to date have clarified the relationship between NRF2 and MSC stemness or interactions between NRF2 and stemness genes. Enhancing NRF2 activity may mimic the effects of hypoxic environments, similar to an in vivo MSC niche in ex vivo cultured MSCs.

Here, we identify NRF2 as a regulator of sirtuin 1 (SIRT1). NRF2 phosphorylation was decreased and NRF2 was exported from the nucleus in late-passage (LP) and differentiated MSCs. The inhibition of NRF2 activity in early-passage (EP) MSCs suppressed the self-renewal and differentiation capacities. NRF2 activation in EP or LP MSCs enhanced the selfrenewal capacity, but suppressed the differentiation potential. NRF2 increased SIRT1 protein expression by decreasing p53 protein levels. RNA interference targeting p53 in NRF2-knockdown MSCs rescued SIRT1 protein levels as well as the self-renewal and differentiation potentials. Our data indicate that NRF2 plays an important role in the maintenance of MSC stemness via p53-SIRT1 regulation.

\section{Results}

NRF2 phosphorylation and activity decreases during long-term cultivation and differentiation in human BM-MSCs. We hypothesized that NRF2 expression or activity decrease during the long-term cultivation or osteogenic differentiation of MSCs. Accordingly, we observed the mRNA and protein levels of NRF2 in EP or LP MSCs as well as in those that were undifferentiated or differentiated to the osteogenic lineage. As shown in Figures 1a and b, the mRNA and protein levels of NRF2 did not change during prolonged cell passage or osteogenic differentiation. The nuclear import of NRF2 is blocked by its dephosphorylation, resulting in a decrease in NRF2 activity. ${ }^{25}$ Consistent with this, we found that the levels of NRF2 phosphorylation decreased during these processes (Figure 1b, upper panel). We also confirmed that the mRNA levels of $\mathrm{HO}-1$ (heme oxygenase-1) and NQO-1 $(N A D(P) H$ dehydrogenase (quinone 1)), which are downstream target genes of NRF2, decreased during these processes (Figures 1c and d). Next, the cellular localization of NRF2 and its phosphorylated form were analyzed by cytosolic and nuclear fractionated western blots and immunocytochemistry. The protein levels of nuclear and phosphorylated NRF2 were nearly detected in LP or differentiated MSCs compared with EP or undifferentiated MSCs (Figures 1e and f). Immunocytochemistry verified the absence of nuclear NRF2 in the LP and differentiated MSCs (Figures $1 \mathrm{~g}$ and $\mathrm{h}$ ). These results indicate that NRF2 phosphorylation and activity may be important factors in the maintenance of the early characteristics of MSCs, whereas the mRNA and protein levels did not change during prolonged cell passages and the osteogenic differentiation process. It is also thought that a decrease in NRF2 activity may be related to loss of EP MSC stemness during these processes. LP MSCs lose their antioxidant ability, which is accompanied by NRF2 dephosphorylation, resulting in higher susceptibility to oxidative stresses than that of EP MSCs.

Regulation of NRF2 activity affects the self-renewal and osteogenic differentiation potentials of EP MSCs. To confirm whether NRF2 activity affects the self-renewal and differentiation potentials of MSCs, ochratoxin A (OTA), which is a NRF2 inhibitor, ${ }^{26}$ was used to suppress NRF2 activity in EP MSCs. Treatment with $10 \mu \mathrm{M}$ OTA successfully suppressed the nuclear import of NRF2 and its phosphorylated form (Figure 2a). Immunofluorescence also showed the suppression of nuclear import (Figure 2b), and the mRNA levels of HO-1 and NQO-1 decreased in response to OTA treatment (Figure 2c). To assess the effects of OTA on the self-renewal capacity of EP MSCs, proliferation and colonyforming unit fibroblast (CFU-F) assays were performed. There was no difference in the proliferation rates between control EP MSCs and OTA-treated EP MSCs at day 3, but the gap between the two groups gradually widened up to day 7 (Figure 2d). OTA significantly reduced the colony-forming ability of EP MSCs cultured for 12 days (Figure 2e) and blocked the ability to differentiate into the osteogenic lineage (Figure 2f). To exclude the nonspecific effects of the compounds, small interfering RNA (siRNA) knockdown of NRF2 and its effects on self-renewal and differentiation of EP MSCs were evaluated, thus resulting in the same effects as OTA treatment (Supplementary Figure S1). These results showed that the selfrenewal and differentiation ability of EP MSCs are inhibited by blocking the nuclear import and phosphorylation of NRF2.

tert-Butylhydroquinone ( $\mathrm{t}-\mathrm{BHQ}$ ) is an activator of NRF2 and regulates NRF2 stabilization by preventing binding with KEAP1. ${ }^{27}$ We treated EP MSCs with $10 \mu \mathrm{M}$ t-BHQ in order to evaluate the self-renewal and osteogenic differentiation potentials. We observed an increase in NRF2 phosphorylation in the nucleus when EP MSCs were exposed to t-BHQ at a dose of $10 \mu \mathrm{M}$ for $12 \mathrm{~h}$ (Figure 3a). It was difficult to observe the difference in the nucleic NRF2 protein levels between control EP MSCs and t-BHQ-treated EP MSCs using immunofluorescence because nucleic NRF2 was generally abundant (Figure 3b). Nevertheless, the mRNA levels of $\mathrm{HO}-1$ and NQO-1 were significantly higher in t-BHQ-treated EP MSCs than in control cells (Figure $3 \mathrm{c}$ ). t-BHQ enhanced the proliferation rate and increased the number of colony-forming cells in t-BHQ-treated EP-MSCs compared with control EP-MSCs, as expected (Figures $3 d$ and e). Unexpectedly, the osteogenic differentiation of EP MSCs was blocked by $10 \mu \mathrm{M}$ t-BHQ (Figure 3f). In general, pluripotent or stemness genes, such as SOX2, NANOG, and OCT3/4, enable the maintenance of self-renewal and undifferentiated states in stem cells. ${ }^{28}$ Therefore, we inferred that the inhibition of osteogenic differentiation caused by NRF2 activation in EP MSCs was due to NRF2-mediated stemness gene regulation.

Enhanced NRF2 activity maintains the undifferentiated state of EP MSCs by increasing SIRT1 expression at the mRNA and protein levels. Three groups were examined. The first group (GROUP I) was EP-MSCs grown for 10 days in osteogenic induction medium with each vehicle for OTA or 
a

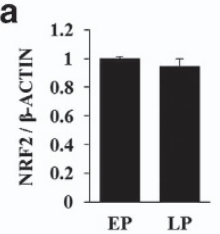

c

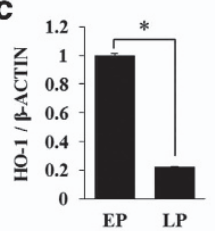

e

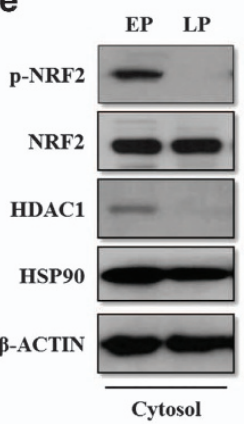

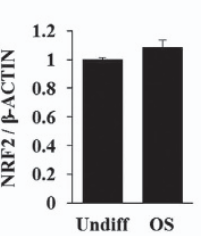
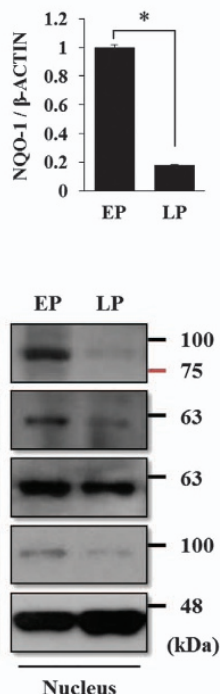

b

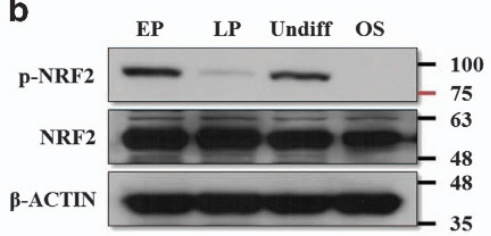

d

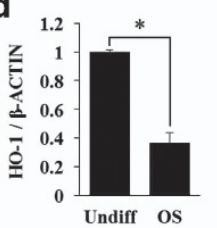

f

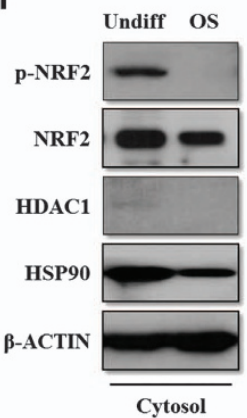

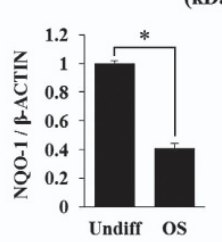

(kDa)

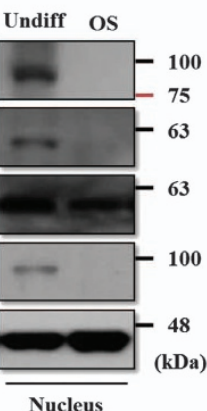

g

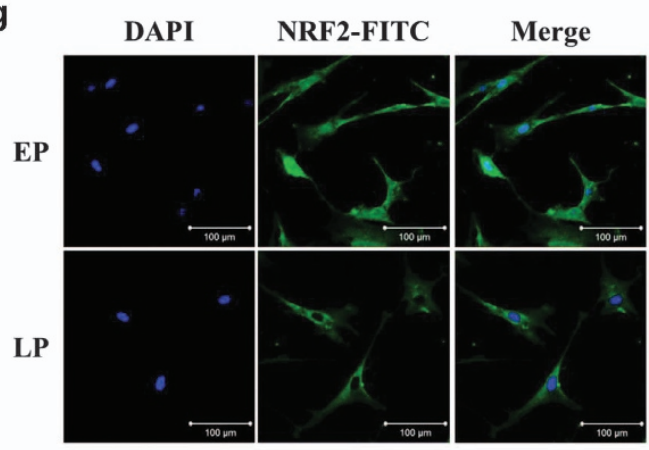

h

DAPI

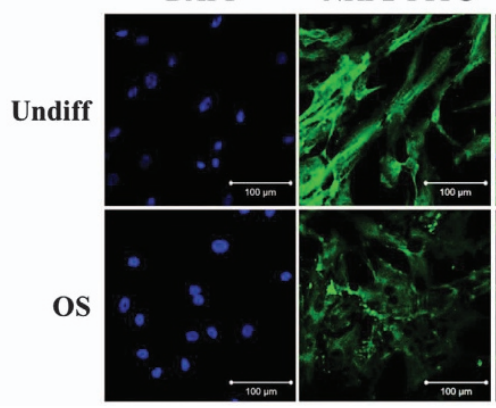

Merge

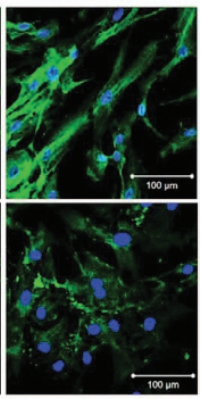

Figure 1 The phosphorylated level and nuclear localization of NRF2 decrease during prolonged cell passages and osteogenic differentiation. (a) EP, LP, undifferentiated, or differentiated MSCs to the osteogenic lineage were harvested at each stage to prepare cell lysates. The mRNA expression of NRF2 was analyzed by qRT-PCR, and (b) the protein levels of NRF2 and phosphorylated NRF2 were analyzed by western blot analysis. The expression level of $\beta$-CATENIN was used as a loading control. For the same $\mathrm{cDNA}$, the mRNA expression levels of $\mathrm{HO}-1$ (c) and NQO-1 (d), which are downstream targets of NRF2, were also analyzed by qRT-PCR. ${ }^{\star} P<0.05$ compared with control EP or undifferentiated MSCs. (e) In the same conditions, the cell lysates were prepared and fractionated into nuclear and cytosolic extracts according to the manufacturer's instructions. The protein levels of NRF2 and phosphorylated NRF2 were analyzed by western blot analysis in the EP and LP MSCs (e) or undifferentiated or osteogenic (OS)-differentiated MSCs (f). The protein level of HSP90 was used as a loading control for cytosolic extracts, and the protein level of HDAC1 was used as a loading control for nuclear extracts. Immunofluorescence was performed to observe the nuclear and cytosolic localization of NRF2 in the EP and LP MSCs (g) or undifferentiated or OS-differentiated MSCs ( $h$ ). The nucleus was stained with DAPI, and NRF2 was stained with FITC-conjugated secondary antibody. The images were obtained using confocal microscopy. Scale bar $=100 \mu \mathrm{m}$. EP, early-passage (passages 1-3) MSCs; LP, late-passage (passages 7-10) MSCs; OS, MSCs differentiated to the osteogenic lineage for 10 days; Undiff, undifferentiated MSCs

t-BHQ. The second group (GROUP II) was EP-MSCs grown for 10 days in osteogenic induction medium with OTA or $\mathrm{t}-\mathrm{BHQ}$ at a dose of $10 \mu \mathrm{M}$. The third group (GROUP III) was EP-MSCs grown for 7 days in an osteogenic induction medium with OTA or t-BHQ at a dose of $10 \mu \mathrm{M}$. The cells were grown for an additional 3 days in osteogenic induction medium without OTA or t-BHQ. As shown in Figure $4 a$, OTA-treated EP MSCs did not differentiate to the osteogenic lineage. The removal of OTA on day 7 of differentiation did not rescue the osteogenic potential of EP MSCs. t-BHQ-treated EP MSCs also did not differentiate to the osteogenic lineage. However, the removal of $\mathrm{t}-\mathrm{BHQ}$ on day 7 significantly rescued the osteogenic potential (Figure 4b). Next, we performed quantitative real-time PCR (qRT-PCR) to confirm the regulation of stemness genes by NRF2. As mentioned previously, SOX2, NANOG, and OCT3/4 are very important stemness genes for the maintenance of MSC self-renewal and multipotency, ${ }^{4,29-31}$ and SIRT1 is also thought to regulate MSC stemness. ${ }^{32}$ The regulation of NRF2 activity by OTA or $\mathrm{t}-\mathrm{BHQ}$ treatment in EP MSCs did not affect the mRNA expression of SOX2, OCT4, or NANOG. Among the stemness genes, only SIRT1 expression was significantly affected by OTA or t-BHQ treatment (Figures $4 \mathrm{c}$ and d). We previously reported that among the general stemness genes, SOX2 may be a key factor for maintaining self-renewal and multipotency. Moreover, SOX2 is regulated via post-translational modification such as acetylation in MSCs, and SIRT1, a lysine deacetylase, directly modulates SOX2 through the inhibition of SOX2 degradation via ubiquitination, although the mRNA level of SOX2 is not changed. ${ }^{32}$ Thus, we selected SIRT1 as a candidate gene that may be regulated by NRF2 activity. We examined whether the t-BHQ-mediated interference of osteogenic differentiation was due to the maintenance of the undifferentiated state of EP MSCs by SIRT1. The p-NRF2 and SIRT1 protein levels decreased, whereas the protein level of Runt-related transcription factor 2 (RUNX2), a master transcription factor involved in osteogenic differentiation, increased during the osteogenic differentiation process (GROUP I) (Figure 4e, left). For the sustained OTA treatment during the osteogenic differentiation of EP MSCs (GROUP II), p-NRF2, SIRT1, and RUNX2 were not observed during the differentiation processes (Figure $4 \mathrm{e}$, middle). After the removal of OTA on day 7 of the differentiation process, p-NRF2, SIRT1, and RUNX2 expression levels were not rescued (Figure 4e, right). Sustained $t-B H Q$ treatment maintained the protein levels of p-NRF2 and SIRT1, whereas 
a

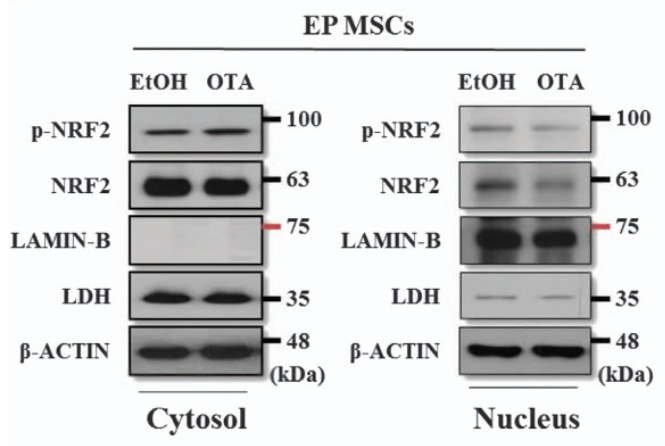

b

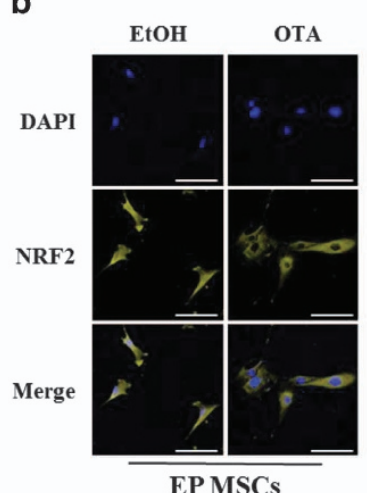

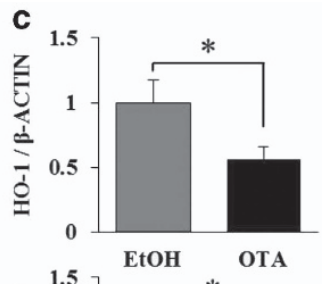

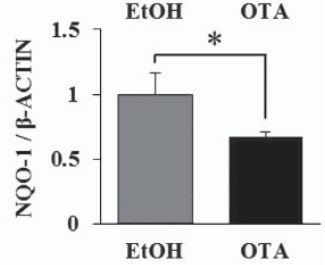

d

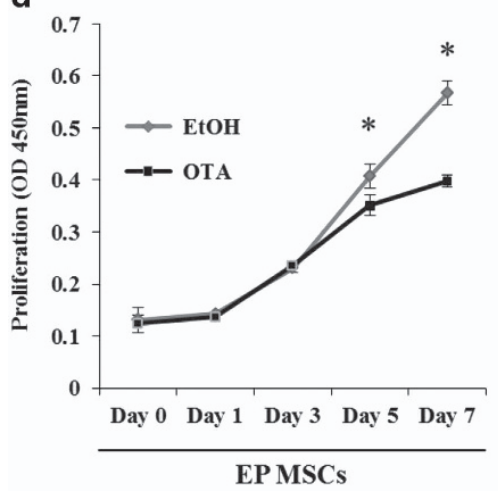

e
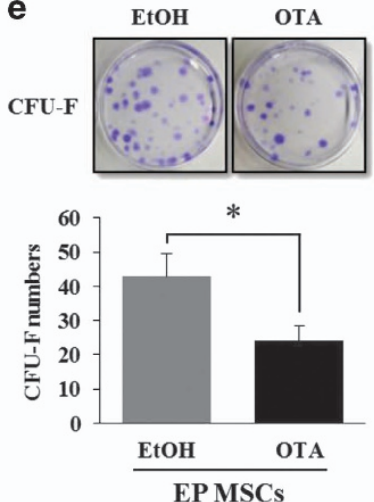

f
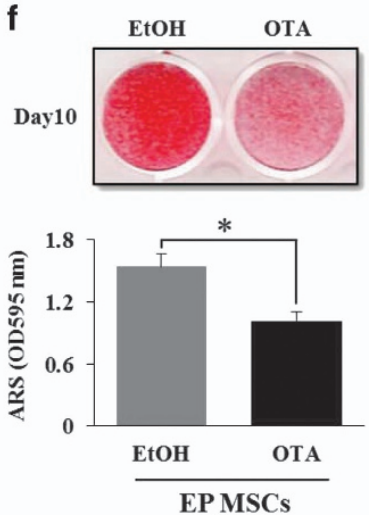

Figure 2 OTA induces nuclear export of NRF2 and decreases the self-renewal capacity and osteogenic differentiation in EP MSCs. (a) EP-MSCs were incubated in basal growth medium (DMEM-LG containing $10 \% \mathrm{FBS}$ ) in the presence of EtOH or OTA (10 $\mu \mathrm{M})$ for $16 \mathrm{~h}$. The cell lysates were prepared for EtOH-treated or OTA-treated EP MSCs, and then fractionated into nuclear and cytosolic extracts. The protein levels of NRF2 and phosphorylated NRF2 were analyzed by western blot analysis for EtOH-treated or OTAtreated EP MSCs. The protein level of LDH was used as a loading control for cytosolic extracts, and the protein level of LAMIN-B was used as a loading control for nuclear extracts. The expression level of $\beta$-CATENIN was also used as a loading control for both cytosolic and nuclear extracts. EtOH was used as a vehicle for OTA. (b) Immunofluorescence was performed to observe the nuclear and cytosolic localization of NRF2 in the EtOH-treated or OTA (10 $\mu \mathrm{M})$-treated EP MSCs. The nuclei were stained with DAPI, and NRF2 was stained with Alexa Fluor 568 (Yellow)-conjugated secondary antibody. The images were obtained using confocal microscopy. Scale bar $=100 \mu \mathrm{m}$. (c) The mRNA expression levels of HO-1 and NQO-1 were also analyzed by qRT-PCR. ${ }^{*} P<0.05$ compared with control EtOH-treated MSCs. (d) A cell proliferation assay was performed to determine the proliferative capacities of EtOH- or OTA (10 $\mu \mathrm{M})$-treated EP MSCs using an EZ-Cytox Kit. Each experiment was performed in triplicate $(n=3)$. (e) EP-MSCs $\left(1 \times 10^{3}\right.$ cells per well in 100-mm dishes) treated with EtOH or OTA $(10 \mu \mathrm{M})$ were incubated in basal growth medium for 12 days. The colony-forming abilities were compared for EP MSCs treated with $\mathrm{EtOH}$ or OTA using crystal violet (CV) staining, and the numbers of colony-forming cells were counted in triplicate by three observers $(n=3)$. ${ }^{*} P<0.05$ compared with EtOHtreated EP MSCs. (f) EP MSCs $\left(8 \times 10^{4}\right.$ cells per well in 12-well plates) treated with EtOH or OTA $(10 \mu \mathrm{M})$ were incubated in osteogenic medium for 10 days. Alizarin red S staining was performed to detect mineral deposition at day 10. For quantitative analysis, absorbance was measured at $595 \mathrm{~nm}$ following destaining with $10 \%$ cetylpyridinium for 30 min. ${ }^{*} P<0.05$ compared with EtOH-treated EP MSCs

the p-NRF2 and SIRT1 protein levels decreased and the RUNX2 protein level increased after the removal of $t-B H Q$ on day 7 of the differentiation process (Figure $4 \mathrm{f})$. These results suggest that NRF2 localization or activity is an important regulator in the SIRT1-mediated maintenance of MSC stemness. In this experiment, the inhibition of NRF2 activity by OTA induced the loss of the self-renewal and osteogenic differentiation potential, whereas NRF2 activation by $\mathrm{t}-\mathrm{BHQ}$ enhanced the self-renewal and osteogenic differentiation potentials of EP MSCs. Therefore, the cellular localization of NRF2 in MSCs may be a crucial factor that determines stem cell characteristics.

p53 is a negative mediator of NRF2-induced SIRT1 stabilization. SIRT1 transcription is negatively regulated by p53 and hypermethylated cancer 1 (HIC1). ${ }^{33}$ HIC1 directly represses SIRT1 transcription via binding the SIRT1 promoter. ${ }^{34}$ The SIRT1 promoter also contains two p53 binding sites, and p53 functions as a transcriptional repressor of SIRT1 ${ }^{35}$ Based on these results, we hypothesized that the decrease in the SIRT1 protein levels by the inhibition of NRF2 activity is due to an increase in HIC1 or p53. NRF2 induces the degradation of tumor-suppressor genes, such as p53 or HIC1, through direct interactions between tumor-suppressor genes and NRF2 downstream target genes. ${ }^{36,37}$ We confirmed that SIRT1 was transcriptionally regulated by the inhibition of activity or the knockdown of NRF2. As shown in Figure $5 \mathrm{a}$, OTA treatment at doses of 1 or $10 \mu \mathrm{M}$ reduced the mRNA levels of HO-1, NQO-1, and SIRT1 dose dependently in EP MSCs. The western blot results also showed that OTA treatment significantly decreased the protein levels of p-NRF2 and SIRT1. In contrast, the p53 protein level, but not HIC1, was highly increased by OTA treatment in EP MSCs (Figure 5b). Using small hairpin RNA (shRNA) 


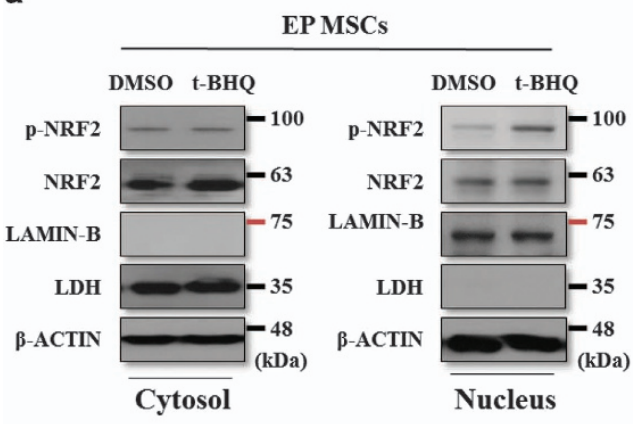

b

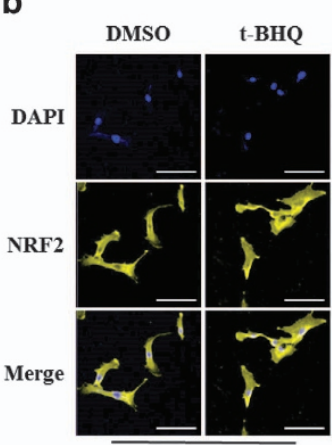

EP MSCs
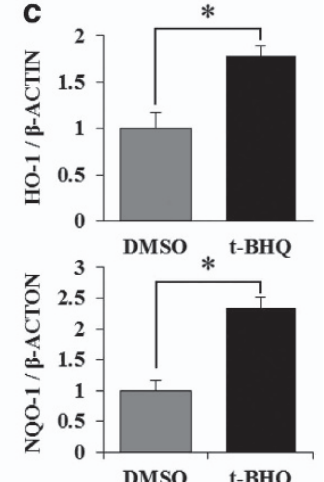

d

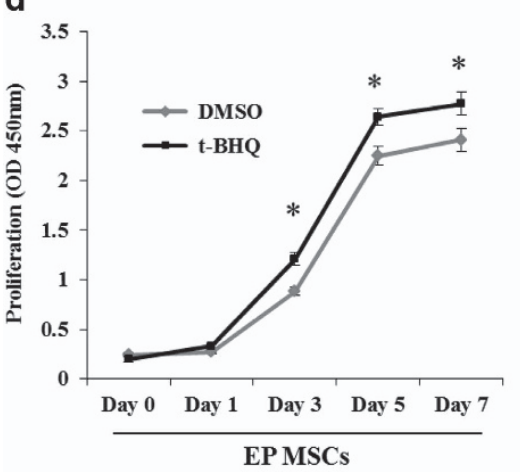

e

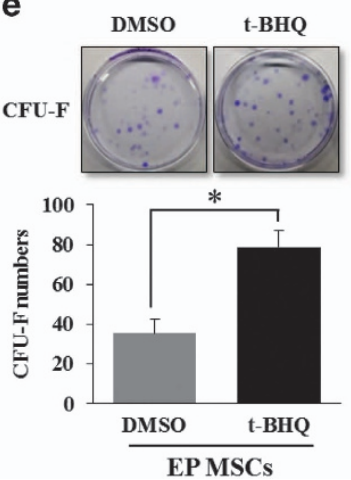

f
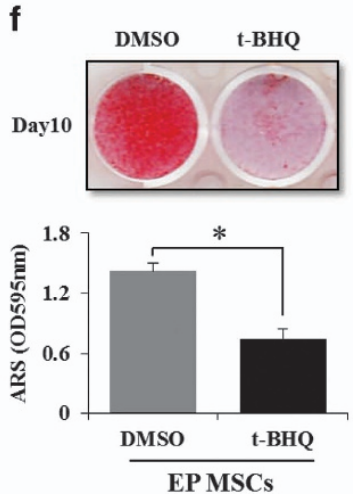

Figure $3 \mathrm{t}-\mathrm{BHQ}$ induces nuclear import of NRF2 and enhances the self-renewal capacity, but does not affect the osteogenic differentiation of EP MSCs. (a) EP MSCs were incubated in basal growth medium (DMEM-LG containing 10\% FBS) in the presence of DMSO or t-BHQ $(10 \mu \mathrm{M})$ for $16 \mathrm{~h}$. Cell lysates were prepared for the DMSO-treated or $\mathrm{t}$-BHQ-treated EP MSCs, and then fractionated into nuclear and cytosolic extracts. The protein levels of NRF2 and phosphorylated NRF2 were analyzed by western blot analysis for the DMSO- or t-BHQ-treated EP MSCs. The LDH protein level was used as a loading control for cytosolic extracts, and the protein level of LAMIN-B was used as a loading control for nuclear extracts. The expression level of $\beta$-CATENIN was also used as a loading control for both cytosolic and nuclear extracts. DMSO was used as a vehicle of t-BHQ. (b) Immunofluorescence was performed to observe the nuclear and cytosolic localization of the NRF2 protein in the DMSO- or t-BHQ (10 $\mu$ M)-treated EP MSCs. The nuclei were stained with DAPI, and NRF2 was stained with Alexa Fluor 568 (Yellow)-conjugated secondary antibody. The images were obtained using confocal microscopy. Scale bar $=100 \mu \mathrm{m}$. (c) The mRNA expression levels of $H O-1$ and NQO-1 were also analyzed with qRT-PCR. ${ }^{*} P<0.05$ compared with control DMSO-treated MSCs. (d) A cell proliferation assay was performed to determine the proliferative capacities of DMSO- or t-BHQ (10 $\mu \mathrm{M})$-treated EP MSCs using an EZ-Cytox Kit. Each experiment was performed in triplicate $(n=3)$. (e) EP-MSCs $\left(1 \times 10^{3}\right.$ cells per well in 100-mm dishes) treated with DMSO or t-BHQ $(10 \mu \mathrm{M})$ were incubated in basal growth medium for 12 days. The colonyforming abilities were compared between EP-MSCs treated with DMSO or t-BHQ using CV staining, and the numbers of colony-forming cells were counted in triplicate by three observers $(n=3) .{ }^{*} P<0.05$ compared with DMSO-treated EP-MSCs. (f) EP-MSCs $\left(8 \times 10^{4}\right.$ cells per well in 12 -well plates) treated with DMSO or t-BHQ $(10 \mu \mathrm{M})$ were incubated in osteogenic medium for 10 days. Alizarin red $\mathrm{S}$ staining was performed to detect mineral deposition at day 10 . For quantitative analysis, absorbance was measured at $595 \mathrm{~nm}$ following destaining with $10 \%$ cetylpyridinium for $30 \mathrm{~min}$. ${ }^{*} P<0.05$ compared with DMSO-treated EP MSCs

targeting NRF2, the mRNA expression levels of $\mathrm{HO}-1$, $N Q O-1$, and SIRT1 decreased significantly in shNRF2infected EP MSCs (Figure 5c). NRF2 knockdown in EP MSCs induced a decrease in the SIRT1 protein level and an increase in the p53 protein level, but did not affect HIC1 (Figure $5 d$ ). These results imply that $p 53$ induced by NRF2 inhibition may suppress the level of SIRT1 expression in EP MSCs. To verify this hypothesis, we purchased siRNA targeting the human $p 53$ or HIC1 genes. In the western blot analysis, OTA decreased the protein levels of p-NRF2 and SIRT1, and increased the p53 protein level. In the same conditions, p53 knockdown using siRNA rescued the SIRT1 protein level, even though the p-NRF2 protein level was still decreased in these OTA-treated EP MSCs (Figure 5e). Knockdown of HIC1 did not affect the SIRT1 protein level in the same conditions (Figure 5f). Knockdown of NRF2 using the shRNA system also successfully decreased the protein levels of NRF2, p-NRF2, and SIRT1, and increased the p53 protein level, but did not affect HIC1. Knockdown of p53 in NRF2-targeting shRNA-infected EP MSCs also rescued the SIRT1 protein level (Figure $5 \mathrm{~h}$ ). These results indicate that p53 is involved in the NRF2-mediated SIRT1 regulation, whereas SIRT1 regulation via HIC1 was not observed. Thus, SIRT1 regulation is predominantly affected by $p 53$, rather than HIC1, in human MSCs.

The self-renewal and osteogenic differentiation potentials suppressed by the inhibition of NRF2 activity can be rescued by p53 RNA interference. We further examined whether targeting p53 would rescue the suppressed selfrenewal and differentiation potentials in OTA-treated or shNRF2-infected EP MSCs. Figure 6a shows that siRNA targeting p53 rescued the colony-forming ability that was decreased in OTA-treated EP MSCs, but this effect was not observed for siRNA targeting HIC1 (Figures 6a and b). As expected, in the shNRF2-infected EP MSCs, siRNA targeting 

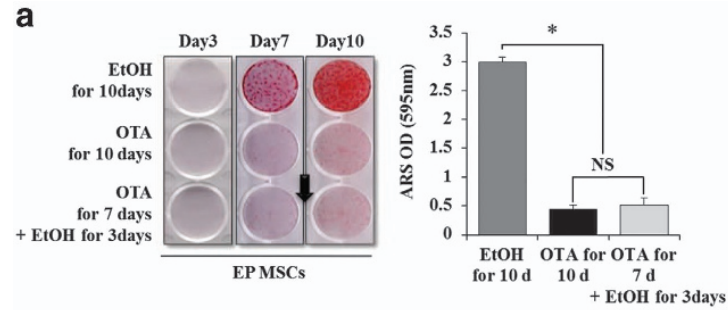

C

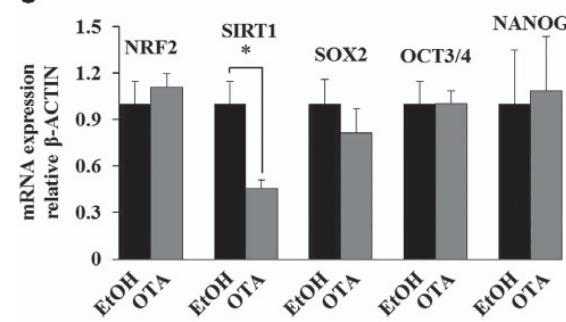

e

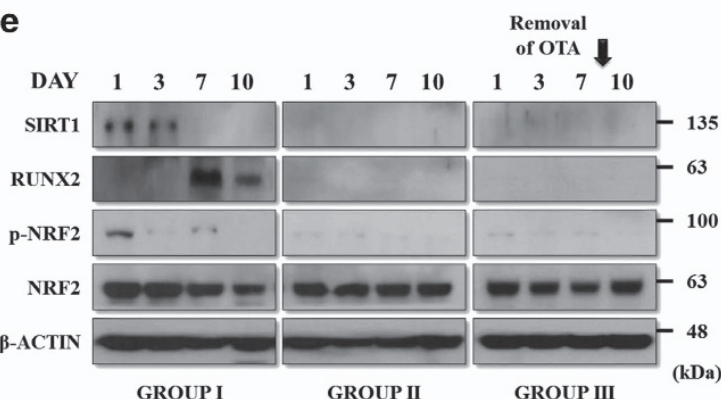

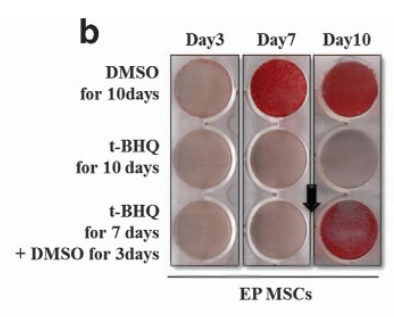

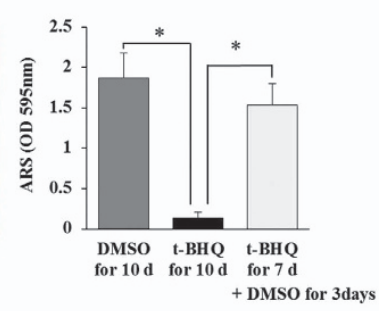

d
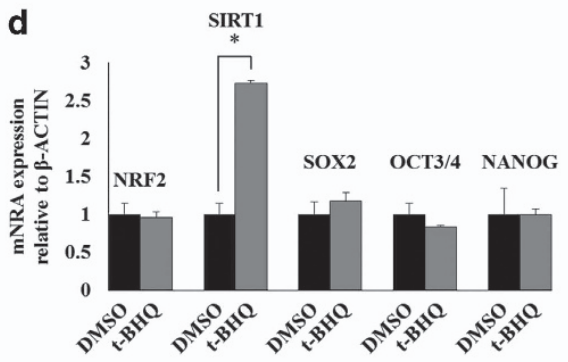

f

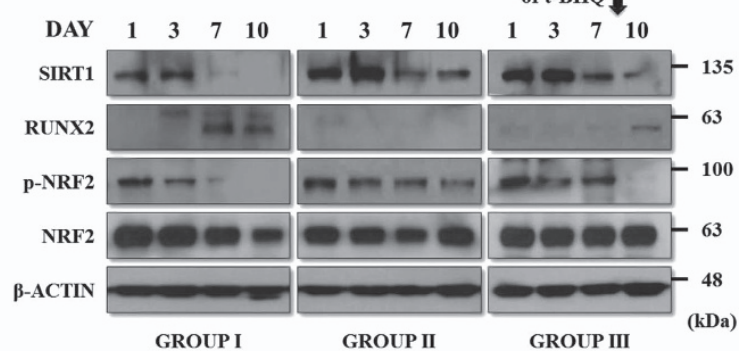

Figure 4 Rescue patterns of suppressed osteogenic potential upon the removal of OTA or t-BHQ, and the association with NRF2 nuclear localization and stemness genes. (a) EP MSCs $\left(8 \times 10^{4}\right.$ cells per well in 12-well plates) treated with EtOH or OTA $(10 \mu \mathrm{M})$ were incubated in osteogenic medium for 10 days. In addition, OTA application was stopped on day 7 of osteogenic differentiation in another group. Alizarin red S staining was performed to detect mineral deposition at days 3,7 , and 10 . For quantitative analysis, absorbance was measured at $595 \mathrm{~nm}$ following destaining with $10 \%$ cetylpyridinium for 30 min. ${ }^{*} P<0.05$ compared with EtOH- or OTA-treated EP MSCs. (b) Similarly, EP MSCs $\left(8 \times 10^{4}\right.$ cells per well in 12-well plates) treated with DMSO or t-BHQ $(10 \mu \mathrm{M})$ were incubated in osteogenic medium for 10 days. In addition, t-BHQ application was stopped on day 7 of osteogenic differentiation in another group. Alizarin red S staining was performed to detect mineral deposition at days 3,7 , and 10 . ${ }^{*} P<0.05$ compared with DMSO- or t-BHQtreated EP MSCs. (c and d) EP MSCs were incubated in basal growth medium (DMEM-LG containing 10\% FBS) in the presence of EtOH/DMSO or OTA (10 $\mu \mathrm{M}) / \mathrm{t}-\mathrm{BHQ}(10 \mu \mathrm{M})$ for $16 \mathrm{~h}$. The mRNA expression levels of NRF2, SIRT1, SOX2, OCT3/4, and NANOG were also analyzed by qRT-PCR. ${ }^{*} P<0.05$ compared with control EtOH/DMSO-treated MSCs. (e and f) EtOH/DMSO or OTA (10 $\mu \mathrm{M}) / \mathrm{t}-\mathrm{BHQ}(10 \mu \mathrm{M})$-treated EP MSCs differentiated to the osteogenic lineage, and cell pellets in each condition were harvested at each stage to prepare cell lysates. The protein levels of NRF2, phosphorylated NRF2, RUNX2, and SIRT1 were analyzed by western blot analysis. The protein level of $\beta$-CATENIN was used as a loading control

p53 significantly enhanced the colony-forming ability that was suppressed by shRNA targeting NRF2, whereas targeting $\mathrm{HIC} 1$ did not affect the self-renewal capacity (Figures $6 \mathrm{c}$ and d). The osteogenic potential suppressed by OTA treatment was also rescued by siRNA targeting p53. Alizarin red $S$ staining showed that suppressed calcification by OTA treatment was rescued in p53-targeting siRNA-transfected EP MSCs (but not in HIC1-targeting siRNA-transfected EP MSCs) (Figures $6 e$ and f). Similarly, in shNRF2-infected EP MSCs, targeting p53 using siRNA yielded the same results to those of the CFU-F assay and alizarin red $S$ staining (Figures $6 \mathrm{~g}$ and $\mathrm{h}$ ). Accordingly, p53 may be an important target in NRF2-mediated SIRT1 regulation for the maintenance of the self-renewal and osteogenic differentiation capacities during prolonged MSC culture.

The self-renewal and osteogenic potentials of LP MSCs can be reactivated by $\mathrm{t}-\mathrm{BHQ}$ treatment. Finally, we tested whether the decreased self-renewal and osteogenic potentials of LP MSCs can be reactivated by inducing the nuclear import of cytosolic NRF2. Application of t-BHQ to LP MSCs increased the mRNA expression of HO-1, NQO-1, and SIRT1, and decreased mRNA expression of p53 (Figures $7 \mathrm{a}$ and $\mathrm{b}$ ). We also found that the p53 protein level was higher in LP-MSCs than EP MSCs, but the HIC1 protein level was not affected by prolonged cell passages. $t-B H Q$ treatment in LP MSCs increased the protein level of $p-N R F 2$, resulting in decreased p53 and increased SIRT1 (Figure 7c). We also confirmed the nuclear localization of NRF2 in t-BHQtreated LP MSCs (Figure 7d). The self-renewal and multipotency of MSCs decreased during prolonged cell passages. Surprisingly, reactivating the NRF2-SIRT1 axis by applying $\mathrm{t}-\mathrm{BHQ}$ to LP MSCs enhanced the colony-forming ability of the cells (Figure 7e). Furthermore, LP MSCs pretreated with $\mathrm{t}-\mathrm{BHQ}$ for $\sim 4-7$ days before osteogenic differentiation were successfully differentiated to the 
a

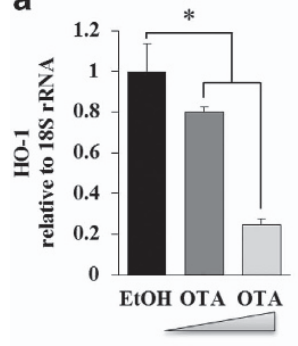

C

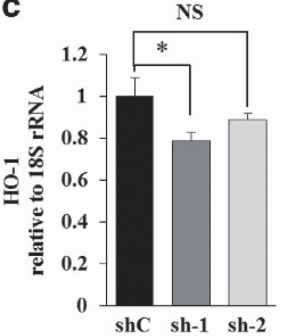

e

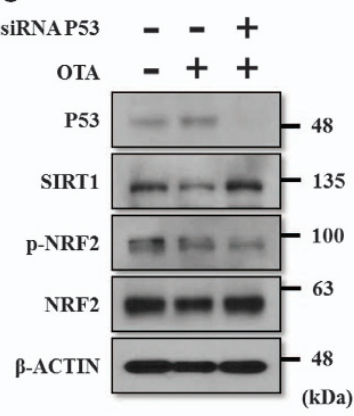

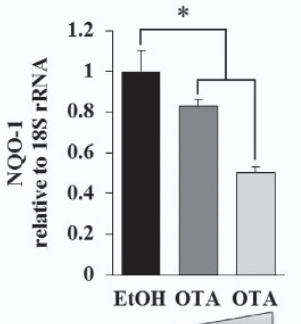
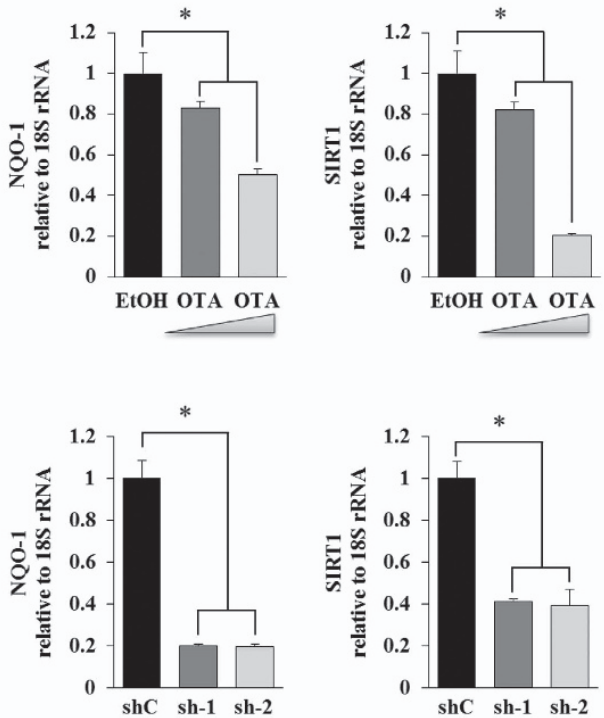

f

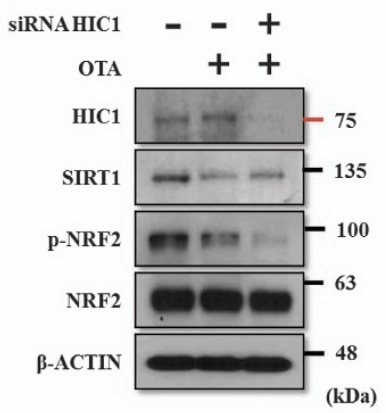

g

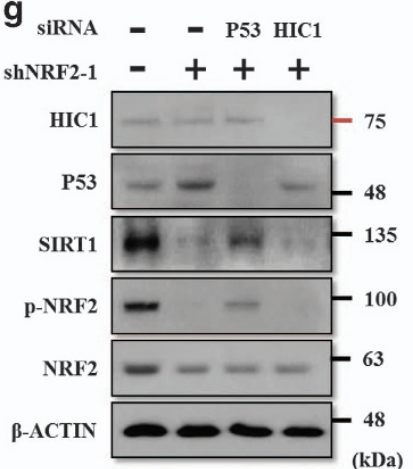

b

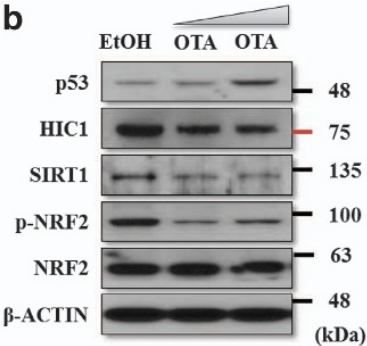

d

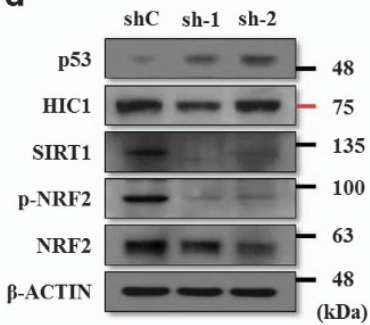

Figure 5 NRF2-mediated SIRT1 regulation occurs in a p53-dependent manner in EP MSCs. (a) EP MSCs were incubated in basal growth medium (DMEM-LG containing $10 \% \mathrm{FBS}$ ) in the presence of EtOH, $1 \mu \mathrm{M}$ OTA, and $10 \mu \mathrm{M}$ OTA for $16 \mathrm{~h}$. The mRNA expression levels of HO-1, NQO-1, and SIRT1 were also analyzed by qRT-PCR. ${ }^{*} P<0.05$ compared with control EtOH-treated MSCs. (b) The protein levels of NRF2, phosphorylated NRF2, SIRT1, HIC1, and p53 were analyzed by western blot analysis. The protein level of $\beta$-CATENIN was used as a loading control. (c) The mRNA expression levels of HO-1, NQO-1, and SIRT1 were also analyzed in EP MSCs infected with nontargeting shRNA, shRNF2-1, or shNRF2-2 by qRT-PCR. ${ }^{*} P<0.05$ compared with nontargeting shRNA-infected MSCs. (d) Similarly, the protein levels of NRF2, phosphorylated NRF2, SIRT1, HIC1, and p53 were analyzed in EP MSCs infected with nontargeting shRNA, shRNF2-1, or shNRF2-2 by western blot analysis. (e) siRNA targeting p53 was transfected in the OTA-treated EP MSCs. The protein levels of NRF2, phosphorylated NRF2, SIRT1, and p53 were analyzed in EtOH-, OTA (10 $\mu$ M)-, or siRNA targeting p53 plus OTA $(10 \mu \mathrm{M})$-treated EP MSCs. (f) siRNA targeting HIC1 was transfected in the OTA-treated EP MSCs. The protein levels of NRF2, phosphorylated NRF2, SIRT1, and HIC1 were analyzed in EtOH-, OTA $(10 \mu \mathrm{M})$-, or siRNA targeting HIC1 plus OTA (10 $\mu \mathrm{M})$-treated EP MSCs. (g) siRNA targeting p53 or HIC1 was transfected with shNRF2-1-infected EP MSCs. The protein levels of NRF2, phosphorylated NRF2, SIRT1, HIC1, and p53 were analyzed in the EP MSCs with nontargeting shRNA, shNRF2-1 alone, shNRF2-1 plus siRNA targeting p53, or shNRF2-1 plus siRNA targeting HIC1. (h) siRNA targeting p53 or HIC1 was transfected with shNRF2-2-infected EP MSCs. The protein levels of NRF2, phosphorylated NRF2, SIRT1, HIC1, and p53 were analyzed in the EP MSCs with nontargeting shRNA, shNRF2-2 alone, shNRF2-2 plus siRNA targeting p53, or shNRF2-2 plus siRNA targeting HIC1. The protein level of $\beta$-CATENIN was used as a loading control. NS, no significance; shC, nontargeting shRNA; sh-1, shNRF2-1; sh-2, shNRF2-2

osteogenic lineage (Figures $7 f$ and $g$ ). These results suggest that the NRF2-SIRT1 axis is an important target in the maintenance of the self-renewal and differentiation potencies of MSCs as well as in rejuvenating long-term MSC cultures in vitro before animal studies and clinical applications.

\section{Discussion}

Our study showed that the inhibition or induction of NRF2 nuclear localization affected cell proliferation and the colonyforming ability of EP MSCs. Interestingly, the in vitro osteogenic potential of MSCs was decreased by treatment with both t-BHQ (a NRF2 activator) and OTA (a NRF2 inhibitor). These results indicate that NRF2 activity is essential for maintaining the self-renewal and osteogenic potentials of MSCs during ex vivo cultivation. Because the sustained nuclear localization of NRF2 also blocked MSC differentiation to the osteogenic lineage, it is possible that sustained nuclear NRF2 maintains an undifferentiated state of MSCs; the undifferentiated state can be maintained in hypoxic environments, mimicked by NRF2/hypoxia-inducible factor-1a (HIF-1a) signaling. ${ }^{12,38}$ Furthermore, oxidative stress induces cellular senescence of human MSCs. ${ }^{39}$ Accordingly, we suggest that sustained NRF2 activation protects MSCs against oxidative stress during ex vivo cultivation, ${ }^{39}$ resulting in the maintenance of MSC stemness. NRF2 negatively regulates osteogenic 
a
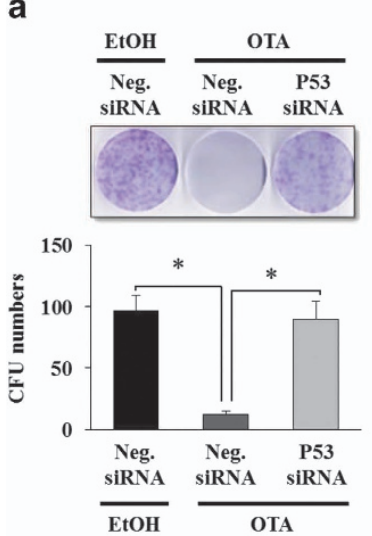

e
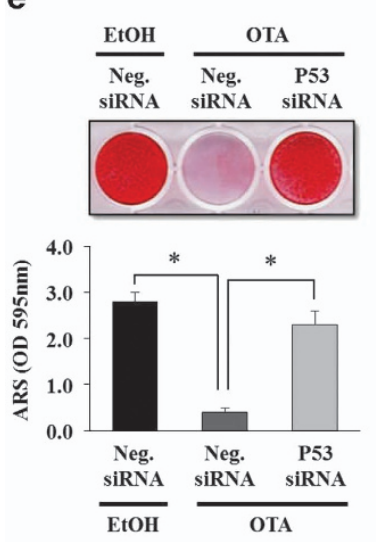
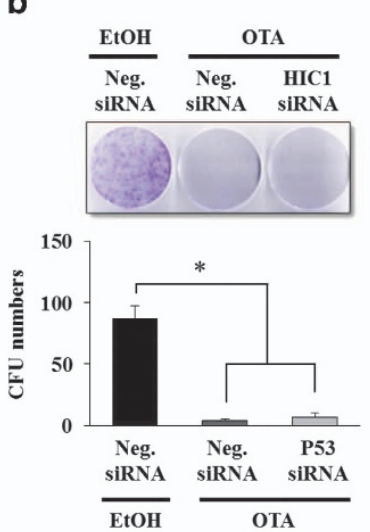

f
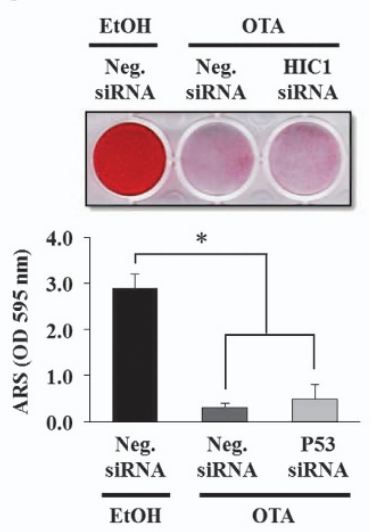

C
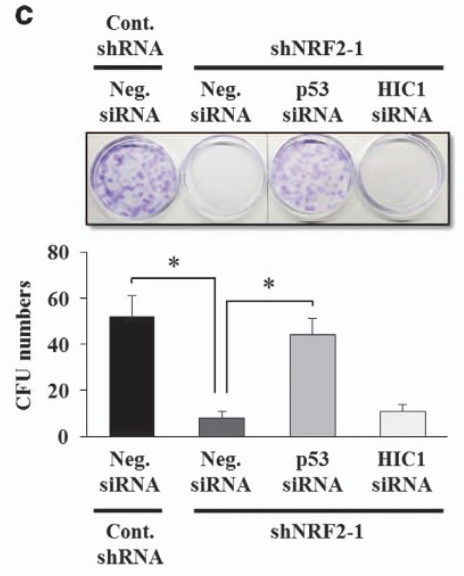

g
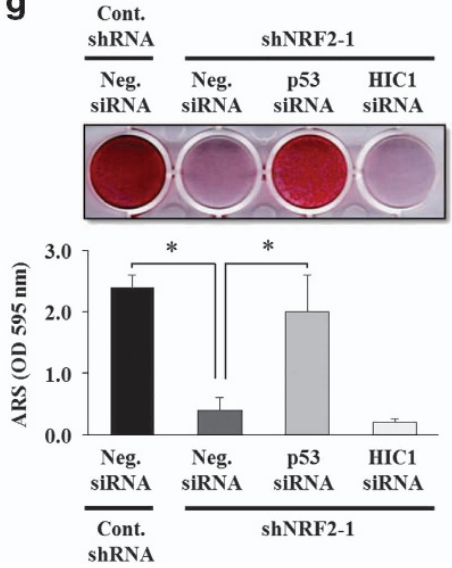

d
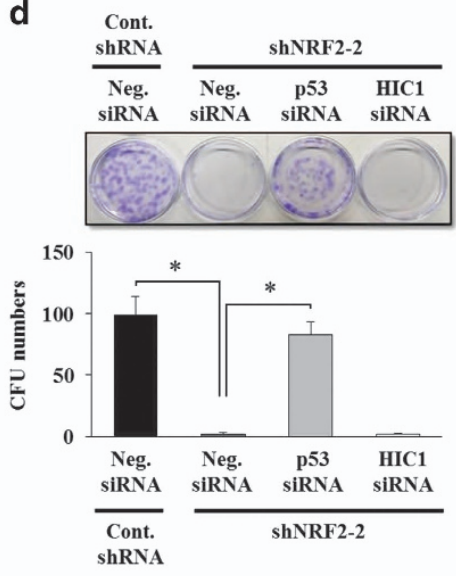

h
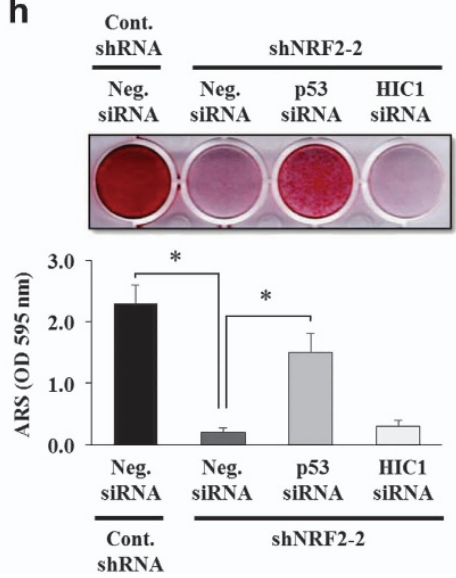

Figure 6 RNA interference of p53 rescues the self-renewal and osteogenic potentials that are suppressed in OTA-treated and shNRF2-infected EP MSCs. The siRNAs targeting p53 (a) or $\mathrm{HIC1}$ (b) were transfected in EP MSCs, and the cells were plated at $1 \times 10^{3}$ cells per well in 100-mm dishes. After $24 \mathrm{~h}$, OTA (10 $\left.\mu \mathrm{M}\right)$ was treated with the siRNA-transfected cells, and the cells were incubated in basal growth medium for 12 days. After 12 days, CV staining was performed, and the numbers of colony-forming cells were counted in triplicate by three observers $(n=4) .{ }^{*} P<0.05$ compared with EtOH-treated EP MSCs transfected with negative siRNA or OTA-treated EP MSCs transfected with negative siRNA. Similarly, the siRNAs targeting p53 or HIC1 were transfected in shNRF2-1 (c)- or shNRF2-2 (d)-infected EP MSCs, and the cells were plated at $1 \times 10^{3}$ cells per well in $100 \mathrm{~mm}$ dishes and incubated in basal growth medium for 12 days. After 12 days, CV staining was performed, and the numbers of colony-forming cells were counted in triplicate by three observers $(n=3) .{ }^{*} P<0.05$ compared with nontargeting shRNA-infected EP MSCs transfected with negative siRNA or shNRF2-infected EP-MSCs transfected with negative siRNA. The siRNAs targeting p53 (e) or HIC1 (f) were transfected in EP MSCs, and the cells were plated at $8 \times 10^{4}$ cells per well in 12-well plates. After $24 \mathrm{~h}$, OTA $(10 \mu \mathrm{M})$ was treated with the siRNA-transfected cells, and the cells were incubated in osteogenic medium for 10 days. After 10 days, alizarin red $\mathrm{S}$ staining was performed to detect mineral deposition. For quantitative analysis, absorbance was measured at $595 \mathrm{~nm}$ following destaining with $10 \%$ cetylpyridinium for 30 min. ${ }^{*} P<0.05$ compared with EtOH-treated EP MSCs transfected with negative siRNA or OTA-treated EP MSCs transfected with negative siRNA $(n=4)$. Similarly, the siRNAs targeting p53 or HIC1 were transfected in shNRF2-1 (g)- or shNRF2-2 (h)-infected EP MSCs, and the cells were incubated in osteogenic medium for 10 days. After 10 days, alizarin red S staining was performed to detect mineral deposition. For quantitative analysis, absorbance was measured at $595 \mathrm{~nm}$ following destaining with $10 \%$ cetylpyridinium for 30 min $(n=3)$. ${ }^{*} P<0.05$ compared with nontargeting shRNA-infected EP MSCs transfected with negative siRNA or shNRF2-infected EP MSCs transfected with negative siRNA. Cont.shRNA, nontargeting shRNA; Neg.siRNA, nontargeting siRNA

differentiation ${ }^{40}$ as well as osteoclastic differentiation. ${ }^{41}$ In addition, there are conflicting results regarding the role of NRF2 in osteogenic differentiation. Human periodontal ligament cells can be efficiently differentiated to the osteogenic lineage by increasing NRF2 levels in nuclear extracts. ${ }^{42}$ NRF2-knockout mice show a significant deficit in postnatal bone acquisition. ${ }^{43}$ Taken together, NRF2 is a key factor in MSC maintenance and osteogenesis; when NRF2 is lacking, MSCs cannot self-renew and differentiate to the osteogenic lineage.

NRF2 also has important roles in the chondrogenic and adipogenic differentiation of MSCs. NRF2 is a negative regulator of cellular differentiation in a chondrocytic cell line,
ATDC5. NRF2 overexpression decreases mRNA expression of type II collagen in ATDC5 cells. ${ }^{44}$ However, sulforaphane (SFN), another NRF2 activator, can suppress gene expression related to osteoarthritis and block cartilage destruction. ${ }^{45}$ The protein level of nuclear Nrf2 also continuously decreases during adipogenic differentiation in ST2 cells, a bone marrowderived MSC cell line. ${ }^{46}$ This expression pattern is required for normal adipocyte differentiation of $3 T 3 L 1$ cells and is associated with increased oxidative stress levels that can facilitate differentiation processes. ${ }^{47}$ SFN can also suppress the adipogenic differentiation of 3 TSL 1 cells. ${ }^{48}$ In contrast, OTA inhibits the adipogenic differentiation of adipose-derived MSCs. ${ }^{49}$ This is consistent with our results regarding the 
a

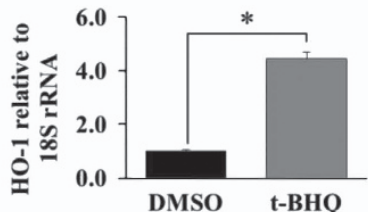

b

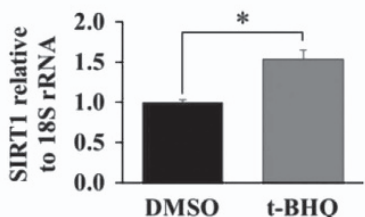

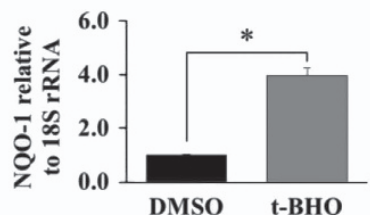

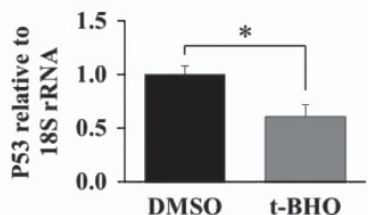

C

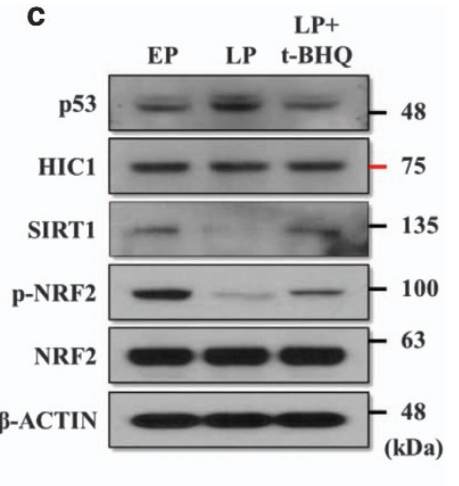

d

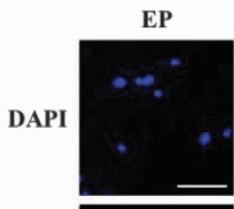

EP

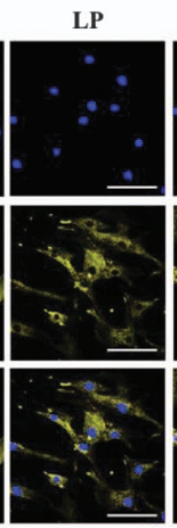

f

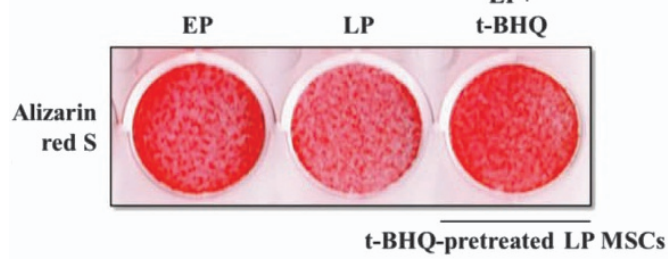

e
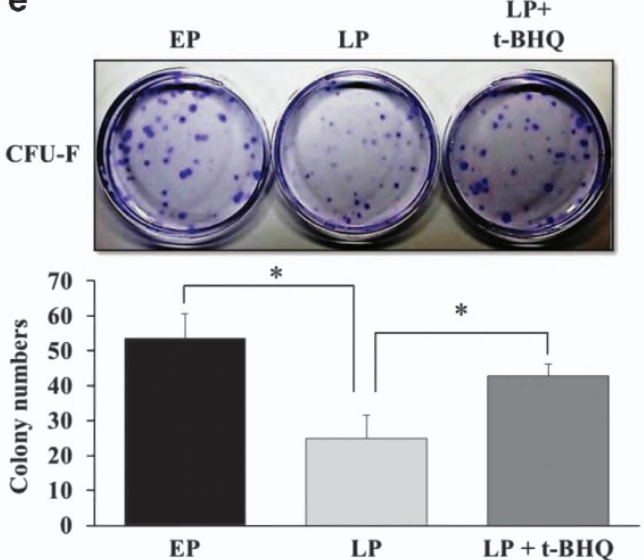

g

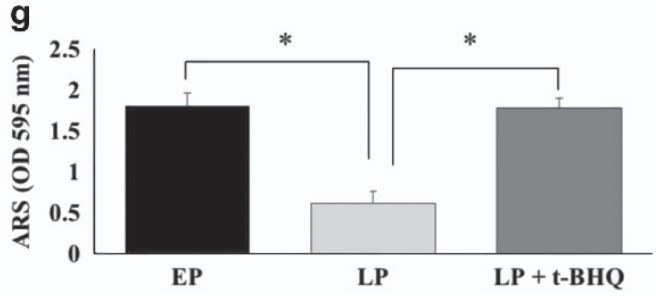

Figure 7 NRF2 activation by t-BHQ enhances the self-renewal capacity and osteogenic potential of LP MSCs. (a) LP MSCs were grown in basal growth medium (DMEM-LG containing $10 \% \mathrm{FBS})$ in the presence of t-BHQ $(10 \mu \mathrm{M})$ for 7 days. The mRNA expression levels of $H O-1, N Q 0-1$, (b) $S I R T 1$, and $p 53$ were also analyzed by qRT-PCR. ${ }^{*} P<0.05$ compared with control EtOH-treated MSCs. (c) Similarly, LP MSCs were grown in basal growth medium in the presence of t-BHQ for 7 days before the western blot analysis. The protein levels of NRF2, phosphorylated NRF2, SIRT1, HIC1, and p53 were analyzed, and the protein level of $\beta$-CATENIN was used as a loading control. (d) Immunofluorescence was performed to observe the nuclear and cytosolic localization of NRF2 in the EP or LP MSCs or t-BHQ- (10 $\mu \mathrm{M})$-pretreated LP MSCs. The nuclei were stained with DAPI, and NRF2 was stained with Alexa Fluor 568 (Yellow)-conjugated secondary antibody. The images were obtained using confocal microscopy. Scale bar $=100 \mu \mathrm{m}$. (e) EP MSCs, LP MSCs, or t-BHQ-pretreated LP MSCs were incubated in basal growth medium for 12 days. The colony-forming abilities were compared using CV staining, and the numbers of colony-forming cells were counted in triplicate by three observers $(n=4)$. ${ }^{*} P<0.05$ compared with EP or LP MSCs. (f) Before the osteogenic differentiation of LP MSCs, LP MSCs were pretreated with t-BHQ. At the starting point of differentiation, LP MSCs were not treated with t-BHQ. EP MSCs, LP MSCs, or t-BHQ-pretreated LP MSCs were plated at $8 \times 10^{4}$ cells per well in 12-well plates and maintained in osteogenic medium for 10 days. After 10 days, alizarin red $S$ staining was performed to detect mineral deposition. (g) For quantitative analysis, absorbance was measured at $595 \mathrm{~nm}$ following destaining with $10 \%$ cetylpyridinium for 30 min. ${ }^{*} P<0.05$ compared with EP or LP MSCs

osteogenic differentiation of MSCs. Taken together, NRF2 may be indispensible for the maintenance of the MSC selfrenewal capacity as well as the multi-differentiation potential to the osteogenic, chondrogenic, and adipogenic lineages.

SIRT1 has been a recent focus of research as a regulator of MSC stemness. ${ }^{50}$ It is required for the long-term cultivation of MSCs, ${ }^{51}$ and its overexpression ameliorates cellular senescence by reversing aged MSCs, resulting in an increase in cell proliferation. $^{52}$ SIRT1 regulates the osteogenic and chondrogenic differentiation of MSCs by deacetylating $\beta$-catenin. ${ }^{53}$ For the maintenance of stemness, SIRT1 positively regulates the self-renewal and multipotency of human MSCs by deacetylating the lysine residue of SOX2. ${ }^{32}$ SIRT1 is known to induce deacetylation of NRF2 that subsequently decreases NRF2dependent gene transcription. ${ }^{54}$ However, it is thought that NRF2 and SIRT1 have similar functions in adult stem cells because the loss of NRF2 in the intestinal stem cells of Drosophila induces ROS levels and age-related degeneration. ${ }^{55}$ Similarly, the loss of SIRT1 is also related to an increase in ROS levels in hematopoietic stem and 


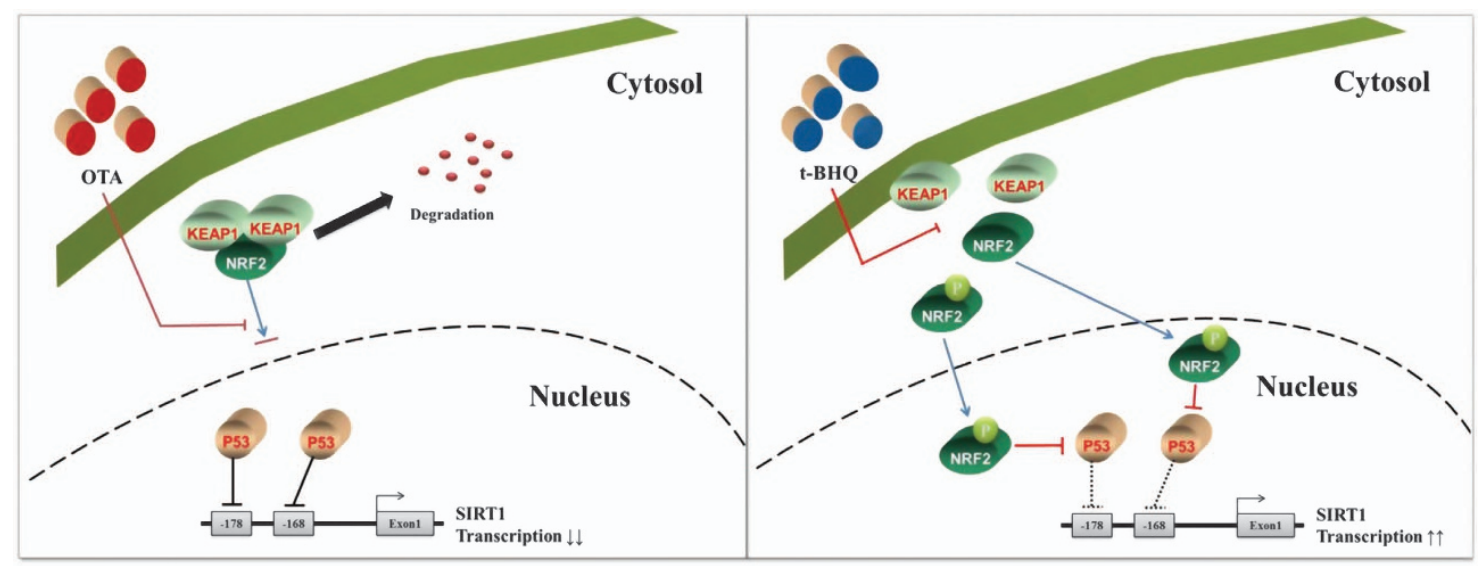

Figure 8 Proposed models of the effects of OTA or t-BHQ on the regulation and maintenance of MSC stemness via SIRT1. Blocking the nuclear import of NRF2 activates p53, which suppresses SIRT1 promoter activity, resulting in a loss of MSC stemness. Conversely, the protein level of p53 can be decreased by the phosphorylation and nuclear import of NRF2, resulting in the activation of SIRT1 transcription as well as the enhancement of MSC stemness

progenitor cells. ${ }^{56}$ These reports indicate that the interaction between NRF2 and SIRT1 in adult stem cells may be involved in defense against oxidative stress during prolonged cell passages, preventing cellular aging and the loss of multipotency. p53 can directly bind to two SIRT1 promoter regions and suppress the transcriptional activity of SIRT $1,{ }^{35}$ whereas NRF2 can induce the degradation of p53 via the regulation of Mdm2 expression. ${ }^{57}$ Based on these results, our study showed that the p53 protein level increased and the SIRT1 protein level decreased in OTA-treated or shNRF2-infected MSCs, and RNA interference of p53 in OTA-treated or shNRF2-infected MSCs rescued the SIRT1 protein level as well as the osteogenic differentiation potential. Furthermore, t-BHQ rejuvenated LP MSCs by enhancing the SIRT1 expression level via NRF2-mediated p53 suppression (Figure 8). Given the importance of these findings in MSCs, additional studies will further increase our understanding of the role of NRF2 in MSC stemness and will facilitate the optimization of the application of NRF2 activators to MSCs.

Recent studies have reported the mechanism of NRF2 action and its new function, excluding its well-known role in antioxidant response. Here, we reviewed the mechanism of NRF2 action that has been reported in several types of stem cells (Supplementary Table S1). In embryonic stem cells, NRF2 acts as a regulator of the proteasome that regulates self-renewal and pluripotency. ${ }^{25}$ In addition, NRF2 can modulate self-renewal and quiescence by regulating CXCR4 in hematopoietic stem cells. ${ }^{58}$ Our study was the first to clarify the molecular mechanisms involved in the regulation of MSCs by NRF2 to maintain the self-renewal and osteogenic potentials via SIRT1. Our findings provide mechanistic insight into how NRF2 contributes to the maintenance of MSC stemness. Thus, the conservation of NRF2 nuclear localization is an important target for the prevention of cellular senescence and the loss of multipotency during prolonged cell passage of ex vivo cultured MSCs.

\section{Materials and Methods}

Cell culture, differentiation, and drug treatment. Bone marrow aspirates were obtained from the posterior iliac crests of seven adult donors, with the approval of the institutional review board (IRB) of the Yonsei University College of Medicine. MSCs isolated from bone marrow were selected based on their ability to adhere to plastic cell culture dishes, and $>98 \%$ of the cultured cells were positive for $\mathrm{CD} 90$ and $\mathrm{CD} 105$, but negative for $\mathrm{CD} 34$ and $\mathrm{CD} 45,{ }^{59}$ as previously described. MSCs were maintained in low-glucose Dulbecco's modified Eagle's medium (DMEM-LG; Invitrogen, Carlsbad, CA, USA) supplemented with $10 \%$ fetal bovine serum (FBS; Gibco, Grand Island, NY, USA) and 1\% antibiotic-antimycotic solution (Invitrogen) at $37^{\circ} \mathrm{C}$ in a $5 \% \mathrm{CO}_{2}$ atmosphere. EP MSCs (passages 1-3) were replated at a density of $5000 \mathrm{cells} / \mathrm{cm}^{2}$, and the cells were subcultured when they were $80 \%$ confluent up to passages 7-10 (LP MSCs). To induce osteogenic differentiation, MSCs were seeded onto 12-well culture pates at a density of $8 \times 10^{4}$ cells per well. The medium used for the osteogenic differentiation of MSCs has been described previously. ${ }^{5}$ Alizarin red $\mathrm{S}$ staining was used to evaluate osteogenic differentiation. Briefly, after cells were fixed in ice-cold $70 \%$ ethanol, freshly prepared $3 \%$ alizarin red S solution (wt/vol) (Sigma, St. Louis, MO, USA) was added, and cells were incubated in the dark for $30 \mathrm{~min}$. For quantitative analysis of alizarin red S, absorbance was detected at $595 \mathrm{~nm}$ following destaining with $10 \%$ cetylpyridinium chloride monohydrate (Sigma) for $20 \mathrm{~min}$. OTA (Sigma) was dissolved in ethanol (EtOH) and used at a concentration of 1-10 $\mu \mathrm{M}$ in EP MSCs. $\mathrm{t}-\mathrm{BHQ}$ (Sigma) was dissolved in dimethyl sulfoxide (DMSO) and used at a concentration of $1-10 \mu \mathrm{M}$ in EP or LP MSCs.

Quantitative real-time PCR (qRT-PCR). Real-time PCR analysis was performed as described previously. ${ }^{32}$ Briefly, total RNA was isolated using an RNeasy Kit (Qiagen, Valencia, CA, USA), according to the manufacturer's instructions. Total RNA $(1 \mu \mathrm{g})$ was reverse-transcribed using the Omniscript Kit (Qiagen). Primer sets were validated and purchased from Bioneer (Daejeon, South Korea; http://www.bioneer.co.kr/). The primers used were as follows: NRF2 (P164742), HO-1 (P133045), NQO-1 (P113225), SIRT1 (P293039), SOX2 (P200205), and NANOG (P255522). There are no validated primers for OCT3/4 (a) or $\beta$-ACTIN. To obtain PCR products specific to OCT3/4(a), which acts as a transcription factor, the primer must include exon 1 in its recognition site; ${ }^{60}$ thus, the following primers were designed: 5'-GCAAGCCCTCATTTCACCA-3' (sense, NM_002701) and 5'-GCCCATCACCTCCACCAC-3' (antisense). The following primers were designed for $\beta$-ACTIN: $5^{\prime}$-GTCCTCTCCCAAGTCCACACA-3' (sense, NM_001101.3) and 5'-GGGCACGAAGGCTCATCATTC-3' (antisense). Mean cycle threshold values from triplicate $(n=3)$ measurements were used to calculate gene expression, with normalization to $\beta$-ACTIN as an internal control.

Western blot analysis. MSCs were lysed in passive lysis buffer (Promega, Madison, WI, USA). Protein concentrations were determined using the Bio-Rad Protein Assay (Bio-Rad Laboratories, Inc., Hercules, CA, USA) and $30 \mathrm{mg}$ of protein was analyzed by $10 \%$ sodium dodecyl sulfate-polyacrylamide gel electrophoresis (SDS-PAGE) (Sigma). Transferred membranes were blocked with $5 \%$ skim milk (BD, Sparks, MD, USA), and incubated for $10 \mathrm{~h}$ with antibodies against NRF2 (Santa Cruz Biotechnology, Santa Cruz, CA, USA), 
phosphorylated-NRF2 (Abcam, Cambridge, UK), HDAC1 (Santa Cruz Biotechnology), HSP90 (Santa Cruz Biotechnology), LAMIN-B (Santa Cruz Biotechnology), LDH (Santa Cruz Biotechnology), RUNX2 (EMD Millipore, San Diego, CA, USA), SIRT1 (Santa Cruz Biotechnology), p53 (Santa Cruz Biotechnology), and HIC1 (Santa Cruz Biotechnology). Membranes were further probed with an antibody against $\beta$-ACTIN (Santa Cruz Biotechnology, Dallas, TX, USA) that served as a loading control.

Nuclear and cytosolic fractionation. EP, LP, undifferentiated, or differentiated MSCs were collected by trypsinization and washed with phosphate-buffered saline (PBS) two times before nuclear and cytosolic fractionation. Nuclear and cytoplasmic fractionation were conducted using the NE-PER Nuclear and Cytoplasmic Extraction Reagents Kit (Thermo Fisher Scientific, Rockford, IL, USA) according to the manufacturer's instructions. Each separated protein was analyzed by western blot analysis.

Immunocytochemistry. EP, LP, undifferentiated, differentiated, OTA-, or $\mathrm{t}-\mathrm{BHQ}$-treated MSCs were seeded at $5000 \mathrm{cells} / \mathrm{cm}^{2}$ on 4-well glass chamber slides (Nalge Nunc International, Rochester, NY, USA), and the cells were incubated in a $5 \% \mathrm{CO}_{2}$ incubator at $37^{\circ} \mathrm{C}$. After an overnight incubation, the cells were washed with PBS followed by fixation with $4 \%$ paraformaldehyde (Sigma) for $30 \mathrm{~min}$. Permeabilization was accomplished with $1 \%$ Triton X-100 in PBS for 10 min followed by blocking for $1 \mathrm{~h}$ with $3 \%$ bovine serum albumin (BSA) in PBS. The cells were incubated with a $1: 200$ dilution of primary antibodies against NRF2 (Santa Cruz Biotechnology) overnight at $4{ }^{\circ} \mathrm{C}$. After washing three times with PBS, the cells were incubated with fluorescein isothiocyanate (FITC) and phycoerythrin-conjugated secondary antibodies (Santa Cruz Biotechnology) or Alexa Fluor 568 (Yellow, Abcam) in a 1:5000 dilution in $3 \%$ BSA-containing PBS for $1 \mathrm{~h}$ at room temperature in the dark. The nuclei were stained with 4,6-diamidino-2-phenyindole (DAPI, Sigma) and then examined using a Zeiss LSM700 scanning laser confocal microscope (Zen 2011; Carl Zeiss Microlmaging GHBH, Jena, Germany).

Proliferation assay. Cell proliferation was examined using an EZ-Cytox Kit (Daeil Lab Service, Seoul, Korea). OTA- or t-BHQ-treated EP-MSCs were seeded in 12-well culture plates at a density of $1 \times 10^{4}$ cells per well. The cells were maintained in DMEM-LG for 7 days, and the cell culture media were replaced once a day during cell viability assay periods. Briefly, after washing cells in PBS, $10 \mu \mathrm{l}$ of EZ-Cytox (tetrazolium salts) solution was added to each well and incubated at $37^{\circ} \mathrm{C}$ for $4 \mathrm{~h}$. After incubation, the conditioned medium was transferred to 96-well plates. The absorbance was measured at $450 \mathrm{~nm}$. All samples were tested in triplicate $(n=6)$

CFU-F assay. EP, LP, OTA-, or t-BHQ-treated MSCs were seeded at $1 \times 10^{3}$ cells in $100 \mathrm{~mm}$ culture dishes, and maintained in DMEM-LG supplemented with $20 \%$ FBS for 10-12 days. OTA-or t-BHQ-treated media were replaced every 2 days during colony formation. Subsequently, the cells were fixed in a $1: 1$ acetone/ methanol fixative, stained with a $20 \%$ crystal violet (CV) solution (Merck, Darmstadt, Germany) for $30 \mathrm{~min}$ in the dark, and washed in distilled water. The colony-forming ability of the stained cells was then evaluated and counted.

Establishment of NRF2-knockdown MSCs. To obtain lentiviral particles with shNRF2, HEK293T cells were seeded in $100 \mathrm{~mm}$ culture dishes at a density of $3 \times 10^{6}$ cells per dish. On the next day, the cells were transfected with lentiviral particles with either a nontargeting shRNA expression plasmid (MISSION Plko.1puro Empty Vector Control Plasmid DNA, Sigma) or two shNRF2 expression plasmids (NFE2L2 MISSION shRNA Stock, TRC numbers: TRCN0000007555 (shNRF-1), TRCN0000007558 (shNRF-2), Sigma) with the Delta 8.9 plasmid (gag, pol, and rev genes) and VSV-G (envelope plasmid, Sigma) using Lipofectamine 2000 (Invitrogen). After $6 \mathrm{~h}$ of transfection, the medium was replaced. The shRNAtransfected HEK293T cells were maintained for 2 days, and then the supernatants were collected and stored at $-70^{\circ} \mathrm{C}$. To knockdown NRF2 in EP-MSCs, the cells were seeded in 6-well plates at a density of $5 \times 10^{4}$ cells per well. After $48 \mathrm{~h}$ of infection, the medium was replaced with freshly prepared medium with $10 \mu \mathrm{g} / \mathrm{ml}$ puromycin dihydrochloride (Sigma), and the cells were maintained for 7 days. The knockdown efficiency of the selected cells was analyzed by western blot analysis. Below is a list of shRNAs targeting NRF2 used in this study.

1. shNRF2-1 (region: 3' UTR)

5'-CCGGGCTCCTACTGTGATGTGAAATCTCGAGATTTCACATCACAGTAGGAG CTTTTT-3

\section{2. shNRF2-2 (region: CDS) 5'-CCGGCCGGCATTTCACTAAACACAACTCGAGTTGTGTTTAGTGAAATGCC GGTTTTT-3'}

siRNA transfection. Scramble, $\mathrm{HIC1}$, and p53 siRNAs were purchased from Bioneer (http://sirna.bioneer.co.kr/). The scramble-sense siRNA targeted the sequence $5^{\prime}$-CCUACGCCACCAAUUUCGU- $3^{\prime}$, and the scramble-antisense siRNA targeted the sequence 5'-ACGAAAUUGGUGGCGUAGG-3'. HIC1-sense siRNA targeted the sequence 5'-AGACGAUGCUGGACACGAU (dTdT)-3', and HIC1-antisense siRNA targeted the sequence 5'-AUCGUGUCCAGCAUCGUCU (dTdT)-3'. p53-sense siRNA targeted the sequence $5^{\prime}$-CACUACAACUACAUGUGUA (dTdT)- 3 ', and p53-antisense siRNA targeted the sequence $5^{\prime}$-UACACAUGUAGUUGUAGUG (dTdT)-3'. NRF2sense siRNA targeted the sequence $5^{\prime}$-GAGACUACCAUGGUUCCAA(dTdT)-3', and NRF2-antisense siRNA targeted the sequence $5^{\prime}$-UUGGAACCAUGGUAGUCUC (dTdT)-3'.

Briefly, EP MSCs treated with OTA $(10 \mu \mathrm{M})$ or infected with shRNA-1 or shRNA2 were plated to obtain $70-80 \%$ confluence in 6-well plates and transfected with $100 \mathrm{nM}$ of HIC1, p53, or scramble (negative control) siRNA using Lipofectamine 2000 (Invitrogen). After $6 \mathrm{~h}$ of transfection, fresh medium was exchanged.

Statistical analysis. Statistical analysis was performed using one-way analysis of variance for multiple comparisons or Student's t-tests for differences between two groups, and the data are expressed as means+S.D. Values of $P<0.05$ were considered statistically significant.

\section{Conflict of Interest}

The authors declare no conflict of interest.

Acknowledgements. This work (NRF-2015R1A2A2A01003876) was supported by the Mid-career Researcher Program through an NRF grant funded by the MEST.

1. Sethe S, Scutt A, Stolzing A. Aging of mesenchymal stem cells. Ageing Res Rev 2006; 5: 91-116.

2. Fehrer C, Lepperdinger G. Mesenchymal stem cell aging. Exp Gerontol 2005; 40: 926-930.

3. Schellenberg A, Lin Q, Schuler H, Koch CM, Joussen S, Denecke B et al. Replicative senescence of mesenchymal stem cells causes DNA-methylation changes which correlate with repressive histone marks. Aging (Albany NY) 2011; 3: 873-888.

4. Yoon DS, Kim YH, Jung HS, Paik S, Lee JW. Importance of Sox2 in maintenance of cell proliferation and multipotency of mesenchymal stem cells in low-density culture. Cell Prolif 2011; 44: 428-440.

5. Yoon DS, Kim YH, Lee S, Lee KM, Park KH, Jang Y et al. Interleukin-6 induces the lineage commitment of bone marrow-derived mesenchymal multipotent cells through downregulation of Sox2 by osteogenic transcription factors. FASEB J 2014; 28: 3273-3286.

6. Zhu W, Chen J, Cong X, Hu S, Chen X. Hypoxia and serum deprivation-induced apoptosis in mesenchymal stem cells. Stem Cells 2006; 24: 416-425.

7. Sart S, Song L, Li Y. Controlling redox status for stem cell survival, expansion, and differentiation. Oxid Med Cell Longev 2015; 2015: 105135

8. Haque N, Rahman MT, Abu Kasim NH, Alabsi AM. Hypoxic culture conditions as a solution for mesenchymal stem cell based regenerative therapy. ScientificWorldJournal 2013; 2013: 632972.

9. Basciano L, Nemos C, Foliguet B, de Isla N, de Carvalho M, Tran N et al. Long term culture of mesenchymal stem cells in hypoxia promotes a genetic program maintaining their undifferentiated and multipotent status. BMC Cell Biol 2011; 12: 12.

10. Ejtehadifar M, Shamsasenjan K, Movassaghpour A, Akbarzadehlaleh P, Dehdilani N, Abbasi $\mathrm{P}$ et al. The effect of hypoxia on mesenchymal stem cell biology. Adv Pharm Bull 2015; 5: 141-149.

11. Lavrentieva A, Majore I, Kasper $C$, Hass R. Effects of hypoxic culture conditions on umbilical cord-derived human mesenchymal stem cells. Cell Commun Signal 2010; 8: 18.

12. Mohyeldin A, Garzon-Muvdi T, Quinones-Hinojosa A. Oxygen in stem cell biology: a critical component of the stem cell niche. Cell Stem Cell 2010; 7: 150-161.

13. Holzwarth C, Vaegler M, Gieseke F, Pfister SM, Handgretinger R, Kerst G et al. Low physiologic oxygen tensions reduce proliferation and differentiation of human multipotent mesenchymal stromal cells. BMC Cell Biol 2010; 11: 11

14. D'Ippolito G, Diabira S, Howard GA, Roos BA, Schiller PC. Low oxygen tension inhibits osteogenic differentiation and enhances stemness of human MIAMI cells. Bone 2006; 39 : 513-522.

15. Fehrer C, Brunauer R, Laschober G, Unterluggauer H, Reitinger S, Kloss $F$ et al. Reduced oxygen tension attenuates differentiation capacity of human mesenchymal stem cells and prolongs their lifespan. Aging Cell 2007; 6: 745-757. 
16. Zhu H, Zhang L, Itoh $\mathrm{K}$, Yamamoto $\mathrm{M}$, Ross D, Trush MA et al. Nrf2 controls bone marrow stromal cell susceptibility to oxidative and electrophilic stress. Free Radic Biol Med 2006; 41: 132-143.

17. Surh YJ, Kundu JK, Na HK. Nrf2 as a master redox switch in turning on the cellular signaling involved in the induction of cytoprotective genes by some chemopreventive phytochemicals. Planta Med 2008; 74: 1526-1539.

18. Itoh K, Chiba T, Takahashi S, Ishii T, Igarashi K, Katoh Y et al. An Nrf2/small Maf heterodimer mediates the induction of phase II detoxifying enzyme genes through antioxidant response elements. Biochem Biophys Res Commun 1997; 236: 313-322.

19. Itoh K, Wakabayashi N, Katoh Y, Ishii T, Igarashi K, Engel JD et al. Keap1 represses nuclear activation of antioxidant responsive elements by Nrf2 through binding to the amino-terminal Neh2 domain. Genes Dev 1999; 13: 76-86.

20. Kobayashi A, Kang Ml, Okawa H, Ohtsuji M, Zenke Y, Chiba T et al. Oxidative stress sensor Keap 1 functions as an adaptor for Cul3-based E3 ligase to regulate proteasomal degradation of Nrf2. Mol Cell Biol 2004; 24: 7130-7139.

21. Yamamoto T, Suzuki T, Kobayashi A, Wakabayashi J, Maher J, Motohashi H et al. Physiological significance of reactive cysteine residues of Keap1 in determining Nrf2 activity. Mol Cell Biol 2008; 28: 2758-2770.

22. Choo KB, Tai L, Hymavathee KS, Wong CY, Nguyen PN, Huang CJ et al. Oxidative stressinduced premature senescence in Wharton's jelly-derived mesenchymal stem cells. Int $\mathrm{J}$ Med Sci 2014; 11: 1201-1207.

23. Mohammadzadeh M, Halabian R, Gharehbaghian A, Amirizadeh N, Jahanian-Najafabadi A, Roushandeh AM et al. Nrf-2 overexpression in mesenchymal stem cells reduces oxidative stress-induced apoptosis and cytotoxicity. Cell Stress Chaperones 2012; 17: 553-565.

24. Jang J, Wang Y, Kim HS, Lalli MA, Kosik KS. Nrf2, a regulator of the proteasome, controls self-renewal and pluripotency in human embryonic stem cells. Stem Cells 2014; 32: 2616-2625.

25. Huang $\mathrm{HC}$, Nguyen T, Pickett $\mathrm{CB}$. Regulation of the antioxidant response element by protein kinase C-mediated phosphorylation of NF-E2-related factor 2. Proc Natl Acad Sci USA 2000; 97: $12475-12480$.

26. Limonciel A, Jennings P. A review of the evidence that ochratoxin A is an Nrf2 inhibitor: implications for nephrotoxicity and renal carcinogenicity. Toxins (Basel) 2014; 6: 371-379.

27. Li J, Johnson D, Calkins M, Wright L, Svendsen C, Johnson J. Stabilization of Nrf2 by tBHQ confers protection against oxidative stress-induced cell death in human neural stem cells. Toxicol Sci 2005; 83: 313-328.

28. Boiani M, Scholer HR. Regulatory networks in embryo-derived pluripotent stem cells. Nat Rev Mol Cell Biol 2005; 6: 872-884.

29. Park SB, Seo KW, So AY, Seo MS, Yu KR, Kang SK et al. SOX2 has a crucial role in the lineage determination and proliferation of mesenchymal stem cells through Dickkopf-1 and c-MYC. Cell Death Differ 2012; 19: 534-545.

30. Pierantozzi E, Gava B, Manini I, Roviello F, Marotta G, Chiavarelli M et al. Pluripotency regulators in human mesenchymal stem cells: expression of NANOG but not of OCT-4 and SOX-2. Stem Cells Dev 2011; 20: 915-923.

31. Tsai CC, Su PF, Huang YF, Yew TL, Hung SC. Oct4 and Nanog directly regulate Dnmt1 to maintain self-renewal and undifferentiated state in mesenchymal stem cells. Mol Cell 2012; 47: 169-182.

32. Yoon DS, Choi Y, Jang Y, Lee M, Choi WJ, Kim SH et al. SIRT1 directly regulates SOX2 to maintain self-renewal and multipotency in bone marrow-derived mesenchymal stem cells. Stem Cells 2014; 32: 3219-3231.

33. Kwon HS, Ott M. The ups and downs of SIRT1. Trends Biochem Sci 2008; 33: 517-525.

34. Chen WY, Wang DH, Yen RC, Luo J, Gu W, Baylin SB. Tumor suppressor HIC1 directly regulates SIRT1 to modulate p53-dependent DNA-damage responses. Cell 2005; 123 : 437-448.

35. Nemoto S, Fergusson MM, Finkel T. Nutrient availability regulates SIRT1 through a forkhead-dependent pathway. Science 2004; 306: 2105-2108.

36. Zhu J, Wang H, Fan Y, Lin Y, Zhang L, Ji X et al. Targeting the NF-E2-related factor 2 pathway: a novel strategy for glioblastoma (review). Oncol Rep 2014; 32: 443-450.

37. Rotblat B, Melino G, Knight RA. NRF2 and p53: Januses in cancer? Oncotarget 2012; 3 : $1272-1283$.

38. Ji X, Wang H, Zhu J, Zhu L, Pan H, Li W et al. Knockdown of Nrf2 suppresses glioblastoma angiogenesis by inhibiting hypoxia-induced activation of HIF-1alpha. Int J Cancer 2014; 135: 574-584.

39. Brandl A, Meyer M, Bechmann V, Nerlich M, Angele P. Oxidative stress induces senescence in human mesenchymal stem cells. Exp Cell Res 2011; 317: 1541-1547.

40. Hinoi E, Fujimori S, Wang L, Hojo H, Uno K, Yoneda Y. Nrf2 negatively regulates osteoblast differentiation via interfering with Runx2-dependent transcriptional activation. J Biol Chem 2006; 281: 18015-18024.
41. Park CK, Lee Y, Kim KH, Lee ZH, Joo M, Kim HH. Nrf2 is a novel regulator of bone acquisition. Bone 2014; 63: 36-46.

42. Chung JH, Kim YS, Noh K, Lee YM, Chang SW, Kim EC. Deferoxamine promotes osteoblastic differentiation in human periodontal ligament cells via the nuclear factor erythroid 2-related factor-mediated antioxidant signaling pathway. J Periodontal Res 2014; 49: $563-573$.

43. Kim JH, Singhal V, Biswal S, Thimmulappa RK, DiGirolamo DJ. Nrf2 is required for normal postnatal bone acquisition in mice. Bone Res 2014; 2: 14033.

44. Hinoi E, Takarada T, Fujimori S, Wang L, lemata M, Uno K et al. Nuclear factor E2 p45related factor 2 negatively regulates chondrogenesis. Bone 2007; 40: 337-344.

45. Davidson RK, Jupp O, de Ferrars R, Kay CD, Culley KL, Norton R et al. Sulforaphane represses matrix-degrading proteases and protects cartilage from destruction in vitro and in vivo. Arthritis Rheum 2013; 65: 3130-3140.

46. Chartoumpekis DV, Ziros PG, Sykiotis GP, Zaravinos A, Psyrogiannis Al, Kyriazopoulou VE et al. Nrf2 activation diminishes during adipocyte differentiation of ST2 cells. Int J Mol Med 2011; 28: 823-828.

47. Vomhof-DeKrey EE, Picklo MJ. NAD(P)H:quinone oxidoreductase 1 activity reduces hypertrophy in 3T3-L1 adipocytes. Free Radic Biol Med 2012; 53: 690-700.

48. Schneider KS, Chan JY. Emerging role of Nrf2 in adipocytes and adipose biology. Adv Nutr 2013; 4: 62-66.

49. Lim S, Jang HJ, Kim JK, Kim JM, Park EH, Yang JH et al. Ochratoxin A inhibits adipogenesis through the extracellular signal-related kinases-peroxisome proliferator-activated receptorgamma pathway in human adipose tissue-derived mesenchymal stem cells. Stem Cells Dev 2011; 20: 415-426.

50. Chen H, Liu X, Chen H, Cao J, Zhang L, Hu X et al. Role of SIRT1 and AMPK in mesenchymal stem cells differentiation. Ageing Res Rev 2014; 13: 55-64.

51. Yuan HF, Zhai $\mathrm{C}$, Yan $\mathrm{XL}$, Zhao DD, Wang JX, Zeng $Q$ et al. SIRT1 is required for long-term growth of human mesenchymal stem cells. J Mol Med (Berl) 2012; 90: 389-400.

52. Chen H, Liu X, Zhu W, Chen H, Hu X, Jiang Z et al. SIRT1 ameliorates age-related senescence of mesenchymal stem cells via modulating telomere shelterin. Front Aging Neurosci 2014; 6: 103

53. Simic P, Zainabadi K, Bell E, Sykes DB, Saez B, Lotinun S et al. SIRT1 regulates differentiation of mesenchymal stem cells by deacetylating beta-catenin. EMBO Mol Med 2013; 5: 430-440.

54. Kawai Y, Garduno L, Theodore M, Yang J, Arinze IJ. Acetylation-deacetylation of the transcription factor Nrf2 (nuclear factor erythroid 2-related factor 2) regulates its transcriptional activity and nucleocytoplasmic localization. J Biol Chem 2011; 286: 7629-7640.

55. Hochmuth CE, Biteau B, Bohmann D, Jasper H. Redox regulation by Keap1 and Nrf2 controls intestinal stem cell proliferation in Drosophila. Cell Stem Cell 2011; 8: 188-199.

56. Rimmele P, Bigarella CL, Liang R, Izac B, Dieguez-Gonzalez R, Barbet G et al. Aging-like phenotype and defective lineage specification in SIRT1-deleted hematopoietic stem and progenitor cells. Stem Cell Reports 2014; 3: 44-59.

57. You A, Nam CW, Wakabayashi N, Yamamoto M, Kensler TW, Kwak MK. Transcription factor Nrf2 maintains the basal expression of Mdm2: an implication of the regulation of p53 signaling by Nrf2. Arch Biochem Biophys 2011; 507: 356-364.

58. Tsai JJ, Dudakov JA, Takahashi K, Shieh JH, Velardi E, Holland AM et al. Nrf2 regulates haematopoietic stem cell function. Nat Cell Biol 2013; 15: 309-316

59. Lee S, Yoon DS, Paik S, Lee KM, Jang Y, Lee JW. microRNA-495 inhibits chondrogenic differentiation in human mesenchymal stem cells by targeting Sox9. Stem Cells Dev 2014; 23: 1798-1808.

60. Liedtke S, Stephan M, Kogler G. Oct4 expression revisited: potential pitfalls for data misinterpretation in stem cell research. Biol Chem 2008; 389: 845-850.

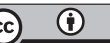

Cell Death and Disease is an open-access journal published by Nature Publishing Group. This work is licensed under a Creative Commons Attribution 4.0 International License. The images or other third party material in this article are included in the article's Creative Commons license, unless indicated otherwise in the credit line; if the material is not included under the Creative Commons license, users will need to obtain permission from the license holder to reproduce the material. To view a copy of this license, visit http://creativecommons.org/licenses/by/4.0/ 